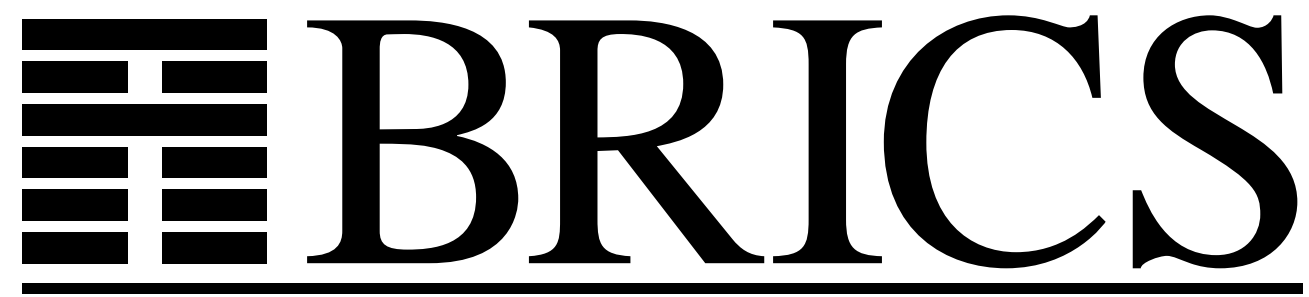

Basic Research in Computer Science

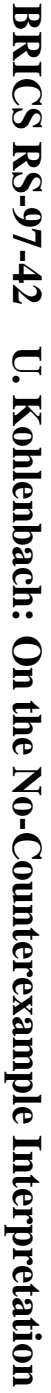

\title{
On the No-Counterexample Interpretation
}

Ulrich Kohlenbach

BRICS Report Series

RS-97-42 
Copyright (c) 1997, $\quad$ BRICS, Department of Computer Science University of Aarhus. All rights reserved.

Reproduction of all or part of this work is permitted for educational or research use on condition that this copyright notice is included in any copy.

See back inner page for a list of recent BRICS Report Series publications. Copies may be obtained by contacting:

\author{
BRICS \\ Department of Computer Science \\ University of Aarhus \\ Ny Munkegade, building 540 \\ DK-8000 Aarhus C \\ Denmark \\ Telephone: +4589423360 \\ Telefax: $\quad+4589423255$ \\ Internet: BRICS@brics.dk
}

BRICS publications are in general accessible through the World Wide Web and anonymous FTP through these URLs:

http://www.brics.dk

ftp: //ftp.brics.dk

This document in subdirectory RS/97/42/ 


\title{
On the no-counterexample interpretation
}

\author{
Ulrich Kohlenbach \\ BRICS* \\ Department of Computer Science \\ University of Aarhus \\ Ny Munkegade, Bldg. 540 \\ DK-8000 Aarhus C, Denmark \\ kohlenb@brics.dk
}

December 1997

\begin{abstract}
In [15],[16] Kreisel introduced the no-counterexample interpretation (n.c.i.) of Peano arithmetic. In particular he proved, using a complicated $\varepsilon$-substitution method (due to W. Ackermann), that for every theorem $A$ ( $A$ prenex) of first-order Peano arithmetic PA one can find ordinal recursive functionals $\underline{\Phi}_{A}$ of order type $<\varepsilon_{0}$ which realize the Herbrand normal form $A^{H}$ of $A$.

Subsequently more perspicuous proofs of this fact via functional interpretation (combined with normalization) and cut-elimination where found. These proofs however do not carry out the n.c.i. as a local proof interpretation and don't respect the modus ponens on the level of the n.c.i. of formulas $A$ and $A \rightarrow B$. Closely related to this phenomenon is the fact that both proofs do not establish the condition $(\delta)$ and - at least not constructively $-(\gamma)$ which are part of the definition of an 'interpretation of a formal system' as formulated in [15].

In this paper we determine the complexity of the n.c.i. of the modus ponens rule for

(i) PA-provable sentences,

(ii) for arbitrary sentences $A, B \in \mathcal{L}(\mathbf{P A})$ uniformly in functionals satisfying the n.c.i. of (prenex normal forms of) $A$ and $A \rightarrow B$, and

(iii) for arbitrary $A, B \in \mathcal{L}(\mathbf{P A})$ pointwise in given $\alpha\left(<\varepsilon_{0}\right)$-recursive functionals satisfying the n.c.i. of $A$ and $A \rightarrow B$.
\end{abstract}

${ }^{*}$ Basic Research in Computer Science, Centre of the Danish National Research Foundation. 
This yields in particular perspicuous proofs of new uniform versions of the conditions $(\gamma),(\delta)$.

Finally we discuss a variant of the concept of an interpretation presented in [17] and show that it is incomparable with the concept studied in [15],[16]. In particular we show that the n.c.i. of $\mathbf{P} \mathbf{A}_{n}$ by $\alpha\left(<\omega_{n}(\omega)\right)$-recursive functionals $(n \geq 1)$ is an interpretation in the sense of [17] but not in the sense of [15] since it violates the condition $(\delta)$.

\section{Introduction}

Let $\exists x A_{0}(x, \underline{a})$ be a $\Sigma_{1}^{0}$-formula in the language $\mathcal{L}(\mathbf{P L})$ of first-order predicate logic $\mathbf{P L}$ $\left(\underline{a}=a_{1}, \ldots, a_{k}\right)$ are all its free variables).

If

$$
\text { PL } \vdash \exists x A_{0}(x, \underline{a})
$$

then by Herbrand's theorem there are terms $t_{1}[\underline{a}], \ldots, t_{n}[\underline{a}]$ (built up out of $\underline{a}$, a distinguished object constant 0 and the object and function constants of $\left.A_{0}\right)^{1}$ such that $\bigvee_{i=1}^{n} A_{0}\left(t_{i}[\underline{a}], \underline{a}\right)$ is a tautology.

This extends to $\Sigma_{n}^{0}$-formulas by introducing so-called index functions. For notational simplicity lets consider $n=4$ only

$$
A(\underline{a}) \equiv \exists x_{1} \forall y_{1} \exists x_{2} \forall y_{2} A_{0}\left(x_{1}, y_{1}, x_{2}, y_{2}, \underline{a}\right) .
$$

We replace $y_{1}, y_{2}$ by $f x_{1}, g x_{1} x_{2}$, where $f, g$ are new function symbols. If $\mathbf{P L} \vdash A$ then

$$
\mathbf{P L} \vdash \exists x_{1}, x_{2} A_{0}\left(x_{1}, f x_{1}, x_{2}, g x_{1} x_{2}, \underline{a}\right)
$$

and so by Herbrand's theorem for $\Sigma_{1}^{0}$-formulas there are terms built up from $\underline{a}, f, g, 0$ and the constants of $A_{0}(x, \underline{a})$ such that

$$
\bigvee_{i=1}^{n} \bigvee_{j=1}^{k} A_{0}\left(t_{i}[\underline{a}, f, g], f\left(t_{i}[\underline{a}, f, g]\right), s_{j}[\underline{a}, f, g], g\left(t_{i}[\underline{a}, f, g], s_{j}[\underline{a}, f, g]\right), \underline{a}\right)
$$

is a tautology.

If we allow definition by cases and characteristic functions for quantifier-free formulas we can avoid the disjunction:

$$
\Phi_{1} \underline{a} f g:=\left\{\begin{array}{l}
t_{1}, \text { if } \bigvee_{j=1}^{k} A_{0}\left(t_{1}, f\left(t_{1}\right), s_{j}, g\left(t_{1}, s_{j}\right), \underline{a}\right) \\
t_{2} \text { if } \neg(\text { case } 1) \wedge \bigvee_{j=1}^{k} A_{0}\left(t_{2}, \ldots\right) \\
\vdots
\end{array}\right.
$$

${ }^{1}$ Throughout this paper $A_{0}, B_{0}, C_{0}, \ldots$ denote quantifier-free formulas. 


$$
\Phi_{2} \underline{a} f g:=\left\{\begin{array}{l}
s_{1}, \text { if } s_{1} \text { if } A_{0}\left(\Phi_{1} \underline{a} f g, f\left(\Phi_{1} \underline{a} f g\right), s_{1}, g\left(\Phi_{1} \underline{a} f g, s_{1}\right), \underline{a}\right) \\
s_{2} \text { if } \neg(\text { case } 1) \wedge A_{0}\left(\Phi_{1} \underline{a} f g, f\left(\Phi_{1} \underline{a} f g\right), s_{2}, g\left(\Phi_{1} \underline{a} f g, s_{2}\right), \underline{a}\right) \\
\vdots
\end{array}\right.
$$

Then

$$
(+) \forall \underline{a}, f, g A_{0}\left(\Phi_{1} \underline{a} f g, f\left(\Phi_{1} \underline{a} f g\right), \Phi_{2} \underline{a} f g, g\left(\Phi_{1} \underline{a} f g, \Phi_{2} \underline{a} f g\right), \underline{a}\right)
$$

holds in a suitable extension of PL.

We say (following Kreisel [15]) that $\Phi_{1}, \Phi_{2}$ satisfy the no-counterexample interpretation of $A$ (short: $\Phi_{1}, \Phi_{2}$ n.c.i. $A$ ).

If $A$ is no longer logically true but provable in some first-order theory, e.g. PA, then definition by cases will not be sufficient in general. In the case of PA for instance one needs all $\alpha$-recursive functionals for $\alpha<\varepsilon_{0}$ and these functionals are also sufficient. This was proved firstly in [16] using an $\varepsilon$-substitution procedure based on [1]. ${ }^{2}$ Later Schwichtenberg [25] gave a proof of this result using a form of cut-elimination (due to [30]) instead.

The cut-elimination procedure does not give a local interpretation of proofs, i.e. given proofs of $A$ and $A \rightarrow B$, a realization of the n.c.i. of $B$ is not computed out of given realizations for the n.c.i of $A$ and $A \rightarrow B$ but by a global proof transformation of the proof of $B$ (which in general will cause a non-elementary increase in the length of this proof). ${ }^{3}$

The method of $\varepsilon$-substitution can be used (as indicated in the proof of the condition $(\delta)$, to be discussed below, in [16]) to obtain $\beta\left(<\varepsilon_{0}\right)$-recursive functionals satisfying the n.c.i. of $B$ out of given $\alpha\left(<\varepsilon_{0}\right)$-recursive functionals satisfying the n.c.i. of (prenex normal forms of) $A$ and $A \rightarrow B$. This method however (which again in general has a non-elementary complexity in the logical depth of $A$ ) does not yield a uniform procedure (given by functionals of type level 3 ) which would provide functionals satisfying the n.c.i. of $B$ uniformly in arbitrary functionals satisfying the n.c.i. of $A$ and $A \rightarrow B$.

A third way to prove the no-counterexample interpretation of PA (by functionals which are $\alpha\left(<\varepsilon_{0}\right)$-recursive) is via Gödel's functional interpretation (combined with negative translation) of PA in the calculus $T$ of primitive recursive functionals of finite type (see e.g. [31]). This (combination of negative translation and) functional interpretation is a local

\footnotetext{
${ }^{2} \mathrm{~A}$ formalization of the method of $\varepsilon$-substitution was given by [29] and used in [20](thm.12).

${ }^{3}$ One should also mention here Gödel's discussion of Gentzen's 1936 consistency proof in his amazing 'Vortrag bei Zilsel' from 1938, first published (together with an English translation in [8]). Here Gödel interprets Gentzen's proof in terms of the no-counterexample interpretation and gives a discussion of the modus ponens rule in these terms which emphasizes the fact that this rule is decisive for the ordinal exponentiation indicating even a kind of local treatment of this rule, however without giving any details ([8] pp. 108-110). See also the illuminating remarks in [27].
} 
proof transformation but at the level of the functional interpretation (of the negative translation) of $A$ and $A \rightarrow B$ and not at the level of their n.c.i.: realizing functionals for $\left(B^{\prime}\right)^{D}$ can be obtained uniformly in any realizations of $\left(A^{\prime}\right)^{D}$ and $\left((A \rightarrow B)^{\prime}\right)^{D}$ by a simple typed lambda term (depending only on the logical form of $A$ and $B$ (Here $A^{\prime}$ and $A^{D}$ denote the negative translation and the functional interpretation of $A$ ).

The passage through higher types makes it necessary to use a normalization procedure for $T$ in order to obtain the n.c.i. in terms of $\alpha\left(<\varepsilon_{0}\right)$-recursive functionals rather than type 2 functionals defined in terms of primitive recursion in higher types (see e.g [21],[25]).

Instead of functional interpretation one could also use a combination of (negative translation plus) the Friedman-Dragalin A-translation and a suitable notion of realizability. If one uses here the so-called 'minimal realizability' of [3] one can avoid the use of higher types but the resulting interpretation again is not local at the level of the n.c.i. but only at the level of the 'minimal realizability' interpretation of (the Friedman-Dragalin translation of the negative translation of) $A, A \rightarrow B{ }^{4}$

In this paper we calibrate the complexity of performing the modus ponens rule directly on the level of the n.c.i. without using higher types. It turns out that even for PA-provable sentences $A$ and $A \rightarrow B$ with n.c.i. in $T_{0}$ no fixed subsystem $T_{n}$ of $T$ suffices: ${ }^{5}$ for every $n \in \mathbb{N}$ there are PA-provable sentence $A, B\left(B \in \Pi_{2}^{0}\right)$ and functionals in $T_{0}$ satisfying (provably in $\widehat{\mathbf{P A}}^{\omega} \wedge^{6}$ ) the n.c.i. of (arbitrary prenex normal forms of) $A$ and $A \rightarrow B$ such that the n.c.i. of $B$ is not satisfied by any function(al) $\in T_{n}$ (since with $A$ and $A \rightarrow B$ also $B$ is provable in PA, it is clear that the n.c.i. of $B$ can be carried out in $T$ ). So already for PA-provable sentences the modus-ponens-complexity of the n.c.i. is not lower than the complexity of the n.c.i. of the whole theory PA. If $A$ and $A \rightarrow B$ are not assumed to be provable in PA, then even $T$ is not sufficient to solve the n.c.i. of the modus ponens rule (uniformly in functionals satisfying the n.c.i. of the assumptions) but $\widehat{\mathbf{P R}}^{\omega}+B R_{0,1}$ is, where $B R_{0,1}$ is the schema of bar recursion for bar recursion of type 0 (with values of type 1) and $\widehat{\mathbf{P R}}^{\omega}$ are all predicative primitive recursive functionals of finite type (in the sense of [13],[4]).

In special cases we can even solve the n.c.i. of the modus ponens as a unification problem yielding functionals satisfying the n.c.i. of $B$ by unification (not depending on the quantifierfree part of $A, B$ but only on the quantifier-prefix of their prenex normal forms): This is

\footnotetext{
${ }^{4}$ In connection with [3] one should mention that some of the result obtained in this paper by 'minimal realizability' can in fact be derived (sometimes in much stronger form) using only well-known facts from the literature $([31],[24])$, see [14].

${ }^{5} T_{n}$ denotes the fragment of Gödel's $T$ (see [7]) with $R_{\rho}$ for $\operatorname{deg}(\rho) \leq n$ only.

${ }^{6} \widehat{\mathbf{P A}}^{\omega} \uparrow$ is the subsystem of $\mathbf{P A}^{\omega}$ based on $T_{0}$ instead of $T$ and with quantifier-free induction only, see [4] and section 2 below.
} 
true for $A \in \Pi_{3}^{0}$ and $B \in \Pi_{\infty}^{0}$ (but already in this case $T$ is not sufficient). This particular matter will be studied further in a subsequent paper.

Kreisel introduced his n.c.i. of arithmetic as an instance of his general definition of an 'interpretation of a system $\Sigma$ ' which we recall here from [15]:

'A computable function $f(n, a)$ is called an interpretation of the system $\Sigma$ if

( $\alpha) f(n, a)$ is the number of a free variable formula $A_{n}$ when $a$ is the number of a formula $\mathcal{A}$ of $\Sigma$ (some Gödel numbering being assumed),

( $\beta$ ) if $\mathcal{A}$ is proved in $\Sigma$, from the proof we find an $\bar{n}$ such that $A_{\bar{n}}$ is verifiable,

$(\gamma)$ if $\neg \mathcal{A}$ is proved in $\Sigma$, for each $n$ we find a substitution for the (individual and function) variables of $A_{n}$ which makes $A_{n}$ false,

$(\delta)$ if $\mathcal{B}$ is proved from $\mathcal{A}$ in $\Sigma$, we find a $g(n)$ so that $B_{g(n)}$ is verifiable if $A_{n}$ is verifiable.'

For the n.c.i. of PA by $\alpha\left(<\varepsilon_{0}\right)$-recursive functionals (resp. functionals in $T$ ) condition $(\alpha)$ follows immediately from the fact that the resulting set of free variable formulas is recursively enumerable. Condition $(\beta)$ follows from each of the proof-methods discussed above. The condition $(\gamma)$ and in particular the condition $(\delta)$ however (which are proved in [16] using the method of $\varepsilon$-substitution) do not follow from the approachs to the n.c.i. by cut-elimination or functional interpretation (or the Friedman-Dragalin translation plus realizability). The condition $(\delta)$ can be formulated in the case of the no-counterexample interpretation of PA in $T$ (or, slightly reformulated, for $\alpha\left(<\varepsilon_{0}\right)$-recursive functionals) as follows

$$
(\delta):\left\{\begin{array}{l}
\text { If } \underline{\Phi}_{A} \text { n.c.i } A \text { is true for } \underline{\Phi}_{A} \in T \text { and } \mathbf{P A} \vdash A \rightarrow B . \\
\text { Then one can construct } \underline{\Phi}_{B} \in T \text { such that } \underline{\Phi}_{B} \text { n.c.i. } B \text { is true. }
\end{array}\right.
$$

Using (a careful analysis of the computational strength of) bar recursion of type 0 we give a new prove of Kreisel's results including a strengthened uniform version of his condition $(\delta)$.

The condition $(\gamma)$ translates in the case of the n.c.i. of PA at hand into

$(\gamma)\left\{\begin{array}{l}\text { If } A \equiv \exists x_{1} \forall y_{1} \ldots \exists x_{k} \forall y_{k} A_{0}\left(x_{1}, y_{1}, \ldots, x_{k}, y_{k}, \underline{a}\right) \in \mathcal{L}(\mathbf{P A}) \text { and PA } \vdash \neg A . \\ \text { Then constructively it holds that for all closed terms } \underline{\Phi} \in T \text { (of suitable types) } \\ \text { there are } \underline{h} \text { such that } A_{0}\left(\Phi_{1} \underline{h}, h_{1}\left(\Phi_{1} \underline{h}\right), \ldots, \Phi_{k} \underline{h}, h_{k}\left(\Phi_{1} \underline{h}, \ldots, \Phi_{k} \underline{h}\right), \underline{a}\right) \text { is false. }\end{array}\right.$ 
Classically the existence of $\underline{h}$ satisfying $(\gamma)$ can be shown quite easily (see remark 4.10). A constructive proof of $(\gamma)$ was given in [16], again by the use of the $\varepsilon$-substitution method. We give a new proof of a uniform strengthening of $(\gamma)$ in section 4.

Finally we discuss a different definition of interpretation presented in [17] and show that this definition is incomparable with the definition given in [15]. In particular we show: the n.c.i. of $\mathbf{P} \mathbf{A}_{n+1}$ (the fragment of $\mathbf{P A}$ with $\Sigma_{n+1}^{0}$-induction only) in $T_{n}$ (which holds by [22]) is an interpretation in the sense of [17] but not in the sense of [15] since the condition $(\delta)$ is violated in this case.

\section{The modus ponens complexity of the no-counterexample interpretation for PA-provable sentences}

Definition 2.1 Let $A: \equiv \exists x_{1} \forall y_{1} \ldots \exists x_{k} \forall y_{k} A_{0}\left(x_{1}, y_{1}, \ldots, x_{k}, y_{k}, \underline{a}\right)^{7}$ be a formula in the language $\mathcal{L}(\mathbf{P A})$ of Peano arithmetic $\mathbf{P A}$ (which for convenience is assumed to contain symbols for every primitive recursive function with the corresponding defining equations as axiom of PA).

The Herbrand normal form $A^{H}$ of $A$ is defined by

$$
A^{H}: \equiv \forall h_{1}, \ldots, h_{k} \exists x_{1}, \ldots, x_{k} \overbrace{A_{0}\left(x_{1}, h_{1} x_{1}, \ldots, x_{k}, h_{k} x_{1} \ldots x_{k}, \underline{a}\right)}^{A_{0}^{H}: \equiv} .
$$

A tuple $\Phi\left(=\Phi_{1}, \ldots, \Phi_{k}\right)$ of functionals of type levels $\leq 2$ satisfies the no-counterexample interpretation of $A$ if $\underline{\Phi} \underline{a} \underline{h}$ realizes ' $\exists \underline{x}$ ' (where $\underline{h}:=h_{1}, \ldots, h_{k}$ and $\underline{x}:=x_{1}, \ldots, x_{k}$ ), i.e. if

$$
\forall \underline{a}, \underline{h} A_{0}\left(\Phi_{1} \underline{a} \underline{h}, h_{1}\left(\Phi_{1} \underline{a} \underline{h}\right), \ldots, \Phi_{k} \underline{a} \underline{h}, h_{k}\left(\Phi_{1} \underline{a} \underline{h}, \ldots, \Phi_{k} \underline{a} \underline{h}\right), \underline{a}\right) .
$$

In this case we write ' $\underline{\Phi}$ n.c.i. $A$ '.

In the following PRA denotes primitive recursive arithmetic extended by classical firstorder predicate logic. $\mathbf{P A}^{\omega}$ (resp. $\mathbf{H A}^{\omega}$ ) is the classical (resp. intuitionistic) arithmetic in all finite types with full induction and all primitive recursive functionals in the sense of Gödel and a quantifier-free rule of extensionality (so in the terminology of [31], $\mathbf{H A}^{\omega}$ is the system WE-HA${ }^{\omega}$ ). $\widehat{\mathbf{P A}}^{\omega} \wedge$ (resp. $\widehat{\mathbf{H A}}^{\omega} \uparrow$ ) denotes the fragment of $\mathbf{P A}^{\omega}$ (resp. $\mathbf{H A}{ }^{\omega}$ ) with quantifier-free induction only and the Gödel-recursors $R_{\rho}$ replaced by the predicative Kleene-recursors $\widehat{R}_{\rho}$ (this systems was introduced and studied in [4]). By $T$ and $\widehat{\mathbf{P R}}^{\omega}$ we denote the quantifier-free parts (in the sense on [31](1.6.13)) of $\mathbf{P A}^{\omega}$ and $\widehat{\mathbf{P A}}^{\omega} \wedge$ respectively.

\footnotetext{
${ }^{7}$ Here $a$ are all the free variables of $A$.
} 
$T_{n}$ is the fragment of $T$ with $R_{\rho}$ for $\rho$ of level $\leq n$ only. $\widehat{\mathbf{P R}}^{\omega}$ is simply a definitorial extension of $T_{0}$ since $R_{0}=\widehat{R}_{0}$ and $\widehat{R}_{\rho}$ for $\rho>0$ is definable from $\widehat{R}_{0}$ by $\lambda$-abstraction.

The type level or degree $\operatorname{deg}(\rho)$ of a type $\rho$ is defined as $\operatorname{deg}(0):=0, \operatorname{deg}(\rho(\tau)):=$ $\max (\operatorname{deg}(\tau)+1, \operatorname{deg}(\rho))$.

Convention: By the phrase 'a functional $\Phi \in T_{(n)}$ ' we always mean 'a closed term $\Phi$ of $T_{(n)}$ '. Sometimes we only write $\Phi \in T_{(n)}$ but again always refer to a closed term of $T_{(n)}$ representing the functional.

Proposition 2.2 For every $n \in \mathbb{N}$ there are sentence (i.e. closed formulas) A, B such that

1) $A$ is prenex,

2) $B \equiv \forall x \exists y B_{0}(x, y) \in \Pi_{2}^{0}$,

3) PRA $\vdash A$,

4) $\mathbf{P A} \vdash A \rightarrow B$,

5) $A$ as well as every prenex normal form $(A \rightarrow B)^{p r}$ of $A \rightarrow B$ has (provably in $\widehat{\mathbf{H A}}^{\omega} \uparrow$ ) a n.c.i. by suitable functionals in $T_{0}$, i.e.

$$
\widehat{\mathbf{H A}}^{\omega} \wedge \vdash \underline{\Phi}_{A} \text { n.c.i. } A \wedge \underline{\Phi}_{(A \rightarrow B)^{p r}} \text { n.c.i. }(A \rightarrow B)^{p r}
$$

with $\underline{\Phi}_{A}, \underline{\Phi}_{(A \rightarrow B)^{p r}} \in T_{0}$,

but:

6) there is no function $\varphi \in T_{n}$ which satisfies the n.c.i. of B, i.e. there is no $\varphi \in T_{n}$ for which $\forall x B_{0}(x, \varphi x)$ is true in the standard model of PA.

Proof: Let $n \in \mathbb{N}$ be fixed. It is well-known that the provably recursive functions of PA are just the $\alpha\left(<\varepsilon_{0}\right)$-recursive functions. Since the definable functions of type 1 in $T_{n}$ are $<\omega_{n+1}(\omega)$-recursive (see [21]), there is a $\Pi_{2}^{0}$-sentence $B \equiv \forall x \exists y B_{0}(x, y)$ in $\mathcal{L}(\mathbf{P A})$ (namely $\forall x \exists y T(\bar{e}, x, y)$ for a certain numeral $\bar{e})$ such that $\mathbf{P A} \vdash B$, but there is no $t^{1} \in T_{n}$ for which $\forall x \in \mathbb{N} B_{0}(x, t x)$ is true.

Since PA $\vdash B$ there are finitely many instances $\tilde{A}_{1}, \ldots, \tilde{A}_{k}$ such that for their universal closures $A_{1}, \ldots, A_{k}$

$$
\text { PRA } \vdash \bigwedge_{i=1}^{k} A_{i} \rightarrow B .
$$


Let $\widehat{A}_{i}(x, \underline{a})$ be the induction formula corresponding to $A_{i}$, where $x$ is the induction variable and $\underline{a}$ includes all parameters, i.e.

$$
A_{i} \leftrightarrow \forall \underline{a}\left(\widehat{A}_{i}(0, \underline{a}) \wedge \forall x\left(\widehat{A}_{i}(x, \underline{a}) \rightarrow \widehat{A}_{i}\left(x^{\prime}, \underline{a}\right)\right) \rightarrow \forall x \widehat{A}_{i}(x, \underline{a})\right) .
$$

We now define

$$
A: \equiv \forall x, \underline{a} \exists y_{1}, \ldots, y_{k} \bigwedge_{i=1}^{k}\left(y_{i}=0 \leftrightarrow \widehat{A}_{i}(x, \underline{a})\right) .
$$

It is clear that

(i) PRA $\vdash A$ (in fact predicate logic with equality plus the axiom $0 \neq S 0$ suffices),

(ii) $\mathbf{P A} \vdash A \rightarrow B$.

In PRA, the variables $x_{1}, \ldots, x_{k}, \underline{a}$ and the variables $y_{1}, \ldots, y_{k}$ can be coded together as single variables $x, y$. Although we do not carry out this coding for the sake of better readability we are free to consider these tuples as single variables from now on. As a consequence we only have to deal with the following prenex normal forms of $A \rightarrow \forall u \exists v B_{0}(u, v)$

$$
\begin{gathered}
\text { (1) } \exists x, \underline{a} \forall u \exists v \forall \underline{y}(\ldots)^{p r}, \\
\text { (2) } \exists x, \underline{a} \forall \underline{y}(\ldots)^{p r}, \\
\text { (3) } \forall u \exists x, \underline{a} \forall \underline{y}(\ldots)^{p r}, \\
\text { (4) } \forall u \exists v, x, \underline{a} \forall \underline{y}(\ldots)^{p r},
\end{gathered}
$$

where $(\ldots)^{p r}$ refer to any prenex normal form of the remaining formula in each case.

For $i=1, \ldots, 4$ the Herbrand normal from $(i)^{H}$ of $(i)$ is implied by the partial Herbrand normal form where Herbrand index functions are introduced only for the universal quantifiers in front of $(\ldots)^{p r}$. So e.g. for (1), (1) ${ }^{H}$ is implied by

$$
(+) \forall f, \underline{g} \exists x, \underline{a}, v([u / f(x, \underline{a})],[\underline{y} / \underline{g}(x, \underline{a}, v)])^{p r} .
$$

One easily shows by classical logic (and $\lambda$-abstraction) that $(+)$ is equivalent to

$$
(*) \exists \underline{g} \forall x, \underline{a}\left(\bigwedge_{i=1}^{k}\left(g_{i} x \underline{a}=0 \leftrightarrow A_{i}(x, \underline{a})\right) \rightarrow \forall u \exists v B_{0}(u, v) .\right.
$$


In fact

$$
\begin{aligned}
& (+) \Leftrightarrow \\
& \forall f, \underline{g}\left(\forall x, \underline{a}, v \bigwedge_{i=1}^{k}\left(g_{i} x \underline{a} v=0 \leftrightarrow \widehat{A}_{i}(x, \underline{a})\right) \rightarrow \exists x, \underline{a}, v B_{0}(f x \underline{a}, v)\right) \Leftrightarrow \\
& \exists \underline{g} \forall x, \underline{a}, v \bigwedge_{i=1}^{k}\left(g_{i} x \underline{a} v=0 \leftrightarrow \widehat{A}_{i}(x, \underline{a})\right) \rightarrow \forall f \exists x, \underline{a}, v B_{0}(f x \underline{a}, v) \Leftrightarrow \\
& \exists \underline{g} \forall x, \underline{a}, v \bigwedge_{i=1}^{k}\left(g_{i} x \underline{a} v=0 \leftrightarrow \widehat{A}_{i}(x, \underline{a})\right) \rightarrow \forall u \exists v B_{0}(u, v) \Leftrightarrow \\
& \exists \underline{g} \forall x, \underline{a} \bigwedge_{i=1}^{k}\left(g_{i} x \underline{a}=0 \leftrightarrow \widehat{A}_{i}(x, \underline{a})\right) \rightarrow \forall u \exists v B_{0}(u, v) .
\end{aligned}
$$

In a similar way one shows the corresponding result for $(2),(3),(4)$. So put together we have

$$
(*) \rightarrow(i)^{H}, \text { where } i=1, \ldots, 4,
$$

by predicate logic (and $\lambda$-abstraction). But $(*)$ and therefore $(i)^{H}$ is provable in $\mathbf{P R A}^{2}$ which is the extension of PRA by adding function quantifiers to PRA and allowing function variables to occur in the schema of quantifier-free induction

$$
\text { QF-IA : } A_{0}(0) \wedge \forall x\left(A_{0}(x) \rightarrow A_{0}\left(x^{\prime}\right)\right) \rightarrow \forall x A_{0}(x) \text {. }
$$

This follows simply by applying QF-IA to $A_{0}(x): \equiv\left(g_{i} x \underline{a}=0\right)$ which yields $A_{i}$ and so $\forall u \exists v B_{0}(u, v)$.

So PRA ${ }^{2} \vdash\left((A \rightarrow B)^{p r}\right)^{H}$ for every prenex normal form of $A \rightarrow B$.

However PRA ${ }^{2}$ has (via negative translation) a functional interpretation and hence a n.c.i. in $\widehat{\mathbf{H A}}^{\omega} \wedge$ by terms $\in T_{0}$. Thus there are functionals $\underline{\Phi}_{(A \rightarrow B)^{p r}} \in T_{0}$ such that

$$
\widehat{\mathbf{H A}}^{\omega} \wedge \vdash \underline{\Phi}_{(A \rightarrow B)^{p r}} \text { n.c.i. }(A \rightarrow B)^{p r}
$$

for each prenex normal form of $A \rightarrow B$. The same holds true for $A$ which is even provable in PRA: there are functionals $\underline{\Phi}_{A} \in T_{0}$ such that

$$
\widehat{\mathbf{H A}}^{\omega} \wedge \vdash \underline{\Phi}_{A} \text { n.c.i. } A \text {, }
$$

which concludes the proof of the proposition.

Remark 2.3 We can replace ' $\varphi \in T_{n}$ ', ' $\underline{\Phi} \in T_{0}$ ' in the proposition above by ' $\varphi$ is $\alpha(<$ $\left.\omega_{n+1}(\omega)\right)$-recursive' and ' $\underline{\Phi}$ is primitive recursive in the sense of Kleene', since the closed terms $t^{2} \in T_{n}$ denote just the $\alpha\left(<\omega_{n+1}(\omega)\right)$-recursive functionals (see e.g. [21]). In the following we only state the $T_{n}$-versions of our results explicitly since it is straightforward to formulate them in terms of ordinal recursive function(al)s as well. 
We now consider the condition $(\delta)$ mentioned in the introduction. This condition was verified for the n.c.i. of PA (by $\alpha\left(<\varepsilon_{0}\right)$-recursive functionals) in [16] using the method of $\varepsilon$-substitution. It does not follow from the proofs of the n.c.i. by cut-elimination or functional interpretation. In section 4 below we will prove a new strong uniform version of this condition.

Let $\mathbf{P A} \mathbf{A}_{n}$ be the subsystem of $\mathbf{P A}$ with induction restricted to $\Sigma_{n}^{0}$-formulas. In [22] it is shown that $\mathbf{P} \mathbf{A}_{n+1}$ has (via negative translation) a functional interpretation in $T_{n}$. Hence also the n.c.i. of $\mathbf{P A}_{n+1}$-provable formulas can be satisfied in $T_{n}$. However as a corollary of proposition 2.2 we have

Corollary 2.4 The no-counterexample interpretation of $\mathbf{P A}_{n+1}$ in $T_{n}$ (or - equivalently by $\alpha\left(<\omega_{n+1}(\omega)\right)$-recursive functionals) does not satisfy the condition $(\delta)$ and hence is not an interpretation in the sense of [15].

Proof: Choose $A, B \in \mathcal{L}\left(\mathbf{P A}_{n+1}\right)$ as in proposition 2.2 and let $(A \rightarrow B)^{p r}$ be any prenex normal form of $A \rightarrow B$ and $\tilde{A}$ be the prenex normal form of $A \wedge(A \rightarrow B)^{p r}$ which results e.g. by shifting first all $A$-quantifiers to the front and then all $(A \rightarrow B)^{p r}$-quantifiers. Already by classical logic, $\tilde{A}$ implies $B$ and so in particular

$$
\mathbf{P A}_{n+1} \vdash \tilde{A} \rightarrow B .
$$

From proposition 2.2 it follows that both $A$ and $(A \rightarrow B)^{p r}$ have a n.c.i. by functionals in $T_{0}$ (i.e. by $\alpha\left(<\omega^{\omega}\right)$-recursive and hence ordinary primitive recursive functionals). From this one easily constructs functionals in $T_{0}$ satisfying the n.c.i. of $\tilde{A}$. However, again by proposition 2.2, $B$ does not have a n.c.i. in $T_{n}$ (and hence not by an $\alpha\left(<\omega_{n+1}(\omega)\right.$ )-recursive function). So $\tilde{A}$ and $B$ provide a counterexample to the condition $(\delta)$ for the n.c.i. of $\mathbf{P A}_{n+1}$ in $T_{n}$.

\section{The uniform modus ponens complexity of the no-counter- example interpretation for arbitrary formulas $A, B \in \mathcal{L}(\mathbf{P A})$}

Definition 3.1 A pair $(\mathcal{T}, \mathcal{F})$ consisting of a theory $\mathcal{T}$ and a quantifier-free functional calculus $\mathcal{F} \subset \mathcal{T}$ suffices for the uniform n.c.i. of the modus ponens rule if for all (prenex) formulas $A, B \in \Pi_{\infty}^{0}\left(A, B \in \mathcal{L}(\mathbf{P A})\right.$ and every prenex normal form $(A \rightarrow B)^{\text {pr }}$ of $A \rightarrow B$ there are functionals $\underline{\Psi} \in \mathcal{F}$ (i.e. closed terms $\underline{\Psi}$ of $\mathcal{F}$ ) such that

$$
\begin{aligned}
\mathcal{T} \vdash \forall \underline{\Phi}_{A}, \underline{\Phi}_{(A \rightarrow B)^{p r}}( & \left(\underline{\Phi}_{A} \text { n.c.i. } A\right) \wedge \\
& \left.\underline{\Phi}_{(A \rightarrow B)^{p r}} \text { n.c.i. }(A \rightarrow B)^{p r}\right) \\
& \left.\rightarrow \underline{\Psi}\left(\underline{\Phi}_{A}, \underline{\Phi}_{(A \rightarrow B)^{p r}}\right) \text { n.c.i. } B\right) .
\end{aligned}
$$


Proposition 3.2 There are sentences $A, B \in \mathcal{L}(\mathbf{P A})$ in prenex normal form such that for all prenex normal forms $(A \rightarrow B)^{p r}$ of $A \rightarrow B$

$\mathbf{P A}^{\omega} \nvdash \exists \underline{\Phi}_{A}\left(\underline{\Phi}_{A}\right.$ n.c.i. $\left.A\right) \wedge \exists \underline{\Phi}_{(A \rightarrow B)^{p r}}\left(\underline{\Phi}_{(A \rightarrow B)^{p r}}\right.$ n.c.i. $\left.(A \rightarrow B)^{p r}\right) \rightarrow \exists \underline{\Phi}_{B}\left(\underline{\Phi}_{B}\right.$ n.c.i. B $)$.

Moreover we can take $A \in \Pi_{3}^{0}$ and $B$ quantifier-free (so that $\left(\underline{\Phi}_{B}\right.$ n.c.i. $\left.B\right) \leftrightarrow B$ with $\underline{\Phi}_{B}$ being the empty tuple).

Proof: Let $A: \equiv \forall x \exists y \forall z(T x x y \vee \neg T x x z)$, where $T$ denotes Kleene's $T$-predicate, and $B: \equiv(0=1)$. There is only one prenex normal form of $A \rightarrow B$ :

$$
\exists x \forall y \exists z(T x x y \vee \neg T x x z \rightarrow 0=1)
$$

and its n.c.i. requires a functionals $\Phi_{1}, \Phi_{2}$ such that

$$
(*) \forall f\left(T\left(\Phi_{1} f, \Phi_{1} f, f\left(\Phi_{1} f\right)\right) \vee \neg T\left(\Phi_{1} f, \Phi_{1} f, \Phi_{2} f\right) \rightarrow 0=1\right) .
$$

The n.c.i. of $A$ is realized by a functional $\Phi_{0}$ such that

$$
(* *) \forall x, g\left(T\left(x, x, \Phi_{0} x g\right) \vee \neg T\left(x, x, g\left(\Phi_{0} x g\right)\right) .\right.
$$

We now show that

$$
\mathbf{P A}^{\omega} \forall \exists \Phi_{0}, \Phi_{1}, \Phi_{2}((*) \wedge(* *)) \rightarrow 0=1 .
$$

We have to show that $\mathbf{P A}^{\omega}+\exists \Phi_{0}, \Phi_{1}, \Phi_{2}((*) \wedge(* *))$ is consistent:

Define $\Phi_{0} x g:=\left\{\begin{array}{l}g 0, \text { if } T(x, x, g 0) \\ 0, \text { otherwise. }\end{array}\right.$

Then one easily verifies that

$$
\mathbf{P A}^{\omega} \vdash \forall x, g\left(T\left(x, x, \Phi_{0} x g\right) \vee \neg T\left(x, x, g\left(\Phi_{0} x g\right)\right) .\right.
$$

Next we show that

$$
\begin{aligned}
\mathbf{P A}^{\omega}+\mathrm{AC}^{1,0} \text {-qf }+\forall f^{1}(f \text { is recursive }) & \vdash \\
& \exists \Phi_{1}, \Phi_{2} \forall f\left(T\left(\Phi_{1} f, \Phi_{1} f, f\left(\Phi_{1} f\right)\right) \vee \neg T\left(\Phi_{1} f, \Phi_{1} f, \Phi_{2} f\right) \rightarrow 0=1\right) .
\end{aligned}
$$

This however follows from the fact that $\mathbf{P A}^{\omega}+\forall f^{1}(f$ is recursive) proves (using the undecidability of the halting problem)

$$
\forall f \exists x, z(T(x, x, f x) \vee \neg T(x, x, z) \rightarrow 0=1),
$$


which implies using $\mathrm{AC}^{1,0}{ }_{-\mathrm{qf}}$

$$
\exists \Phi_{1}, \Phi_{2} \forall f\left(T\left(\Phi_{1} f, \Phi_{1} f, f\left(\Phi_{1} f\right)\right) \vee \neg T\left(\Phi_{1} f, \Phi_{1} f, \Phi_{2} f\right) \rightarrow 0=1\right) .
$$

The proof is now finished by verifying the consistency of $\mathbf{P A}^{\omega}+\mathrm{AC}^{1,0}-\mathrm{qf}+\forall f^{1}(f$ is recursive $)$ which however follows from the fact that

$$
\text { HEO } \models \mathbf{P A}^{\omega}+\mathrm{AC}^{1,0}-\mathrm{qf}+\forall f^{1}(f \text { is recursive }),
$$

where HEO is the type structure of the hereditarily effective operations in all finite types (the fact that $\mathbf{H E O}$ forms a model of $\mathbf{P A}{ }^{\omega}$ is proved in [31]. That it is a model of $\mathrm{AC}^{1,0}{ }_{\text {-qf }}$ follows from the fact that one can always find an effective choice functional by unbounded search since quantifier-free formulas of $\mathbf{P} \mathbf{A}^{\omega}$ are decidable)

Corollary $3.3\left(\mathbf{P A}^{\omega}, T\right)$ does not suffice for the uniform n.c.i. of the modus ponens rule.

Remark 3.4 1) The proof above does not exclude the possibility that e.g.

$\left(\mathbf{P A}^{\omega}+A C_{a r}^{0,0}, T\right)$ satisfies the uniform n.c.i. of the modus ponens rule which remains an open problem. Nevertheless we will show below that even $\left(\mathcal{S}^{\omega}, T\right)$ does not suffice to solve uniformly the unification problem associated with the n.c.i. of the modus ponens (which however does not exclude other ways of satisfying the n.c.i. of the modus ponens)

2) In section 4 below we will show that $\left(\mathbf{P A}^{\omega}, T\right)$ suffices for the pointwise $n . c . i$. of the modus ponens in the sense that one can construct functionals of type level 3 in a genuine extension of $T$ which produce out of given functionals $\in T$ which satisfy the n.c.i. of $A,(A \rightarrow B)^{p r}$ functionals $\in T$ which satisfy the n.c.i. of $B$.

We now show that both $\left(\widehat{\mathbf{H A}}^{\omega} \wedge+\mu, \widehat{\mathbf{P R}}^{\omega}+\mu\right)$ and $\left(\widehat{\mathbf{H A}}^{\omega} \wedge+B R_{0,1}, \widehat{\mathbf{P R}}^{\omega}+B R_{0,1}\right)$ do suffice:

Definition $3.5([4]) \mathcal{T}^{\omega}+\mu$ is the extension of $\mathcal{T}^{\omega}$ obtained by adding a constant $\mu^{2}$ together with the axioms

$$
(\mu): f^{1} x={ }_{0} 0 \rightarrow f(\mu f)={ }_{0} 0 \wedge \mu f \leq_{0} x, f(\mu f) \neq 0 \rightarrow \mu f={ }_{0} 0 .
$$

Definition $3.6([\mathbf{2 8}]) \mathcal{T}^{\omega}+B R_{\rho, \tau}$ is the extension of $\mathcal{T}^{\omega}$ obtained by adding the bar recursor constant $B_{\rho, \tau}$ with the axioms

$$
\left(B R_{\rho, \tau}\right):\left\{\begin{array}{l}
x\left(\overline{y, n^{0}}\right)<n \rightarrow B_{\rho, \tau} x z \text { uny }=_{\tau} z n y \\
x(\overline{y, n}) \geq n \rightarrow B_{\rho, \tau} x z \text { uny }={ }_{\tau} u\left(\lambda D^{\rho} . B_{\rho, \tau} x z u n^{\prime}(\overline{y, n} * D)\right) n y
\end{array}\right.
$$


where $y$ is of type $0(\rho 0)$ and $u$ is of type $\tau(\rho 0)(0)(\tau \rho)$ and

$$
(\overline{y, n} * D)\left(k^{0}\right)=\rho\left\{\begin{array}{l}
y k, \text { if } k<n \\
D, \text { if } k=n \\
0^{\rho}, \text { otherwise }
\end{array}\right.
$$

Proposition 3.7 Let $(\mathcal{T}, \mathcal{F})$ be either $\left(\widehat{\mathbf{H A}}^{\omega} \uparrow+\mu, \widehat{\mathbf{P R}}^{\omega}+\mu\right)$ or $\left(\widehat{\mathbf{H A}}^{\omega} \uparrow+B R_{0,1}, \widehat{\mathbf{P R}}^{\omega}+\right.$ $\left.B R_{0,1}\right)$. Then $(\mathcal{T}, \mathcal{F})$ suffices for the n.c.i. of the modus ponens (uniformly in functionals satisfying the n.c.i. any of prenex normal forms of $A$ and $A \rightarrow B$ ).

Proof: Lets consider the schema of arithmetical choice

$$
\mathrm{AC}_{a r}^{0,0}: \forall x \exists y A(x, y) \rightarrow \exists f \forall x, y A(x, f x),
$$

where $A \in \Pi_{\infty}^{0}$ ( $A$ may contain function parameters).

One easily verifies that

$$
\widehat{\mathbf{P A}}^{\omega} \uparrow+\mathrm{AC}_{a r}^{0,0} \vdash\left(\exists \underline{\Phi}_{A}\left(\underline{\Phi}_{A} \text { n.c.i. } A\right)\right) \rightarrow A
$$

for all prenex formulas $A \in \Pi_{\infty}^{0}$. Since furthermore

$$
\widehat{\mathbf{P A}}^{\omega} \wedge \vdash B \rightarrow B^{H}
$$

for all prenex formulas $B \in \Pi_{\infty}^{0}$ we have

$$
\begin{aligned}
& \widehat{\mathbf{P A}}^{\omega} \wedge+\mathrm{AC}_{a r}^{0,0} \vdash \\
& \left.\quad \exists \underline{\Phi}_{A}\left(\underline{\Phi}_{A} \text { n.c.i. } A\right) \wedge \exists \underline{\Phi}_{(A \rightarrow B)^{p r}}\left(\underline{\Phi}_{(A \rightarrow B)^{p r}} \text { n.c.i. }(A \rightarrow B)^{p r}\right) \rightarrow \forall \underline{h}, \underline{a} \exists \underline{x} B_{0}^{H}(\underline{h}, \underline{x}, \underline{a})\right),
\end{aligned}
$$

where $\forall \underline{h} \exists \underline{x} B_{0}^{H}(\underline{h}, \underline{x}, \underline{a})$ is the Herbrand normal form $B^{H}(\underline{a})$ of $B(\underline{a})$ and $\underline{a}$ are all free variables of $B$.

$\widehat{\mathbf{P A}}^{\omega} \wedge+\mathrm{AC}_{\text {ar }}^{0,0}$ has (via negative translation) a functional interpretation in $\mathcal{T}$ by terms $\in \mathcal{F}$. For $\left(\widehat{\mathbf{H A}}^{\omega} \uparrow+\mu, \widehat{\mathbf{P R}}^{\omega}+\mu\right)$ this is proved in [4]. For $\left(\widehat{\mathbf{H A}}^{\omega} \uparrow+B R_{0,1}, \widehat{\mathbf{P R}}^{\omega}+B R_{0,1}\right)$ this follows from [28] using the facts that $\widehat{\mathbf{P A}}^{\omega} \wedge$ has an interpretation in $\widehat{\mathbf{P R}}^{\omega}$, that $\mathrm{AC}_{a r}^{0,0}$ is derivable in $\widehat{\mathbf{P A}}^{\omega} \uparrow+\Pi_{1}^{0}-\mathrm{AC}^{0,0}$ (note that $\widehat{\mathbf{P A}}^{\omega} \uparrow+\Pi_{1}^{0}-\mathrm{AC}^{0,0} \vdash \Pi_{1}^{0}-\mathrm{CA}$ and so by iteration using the presence of function parameters in $\Pi_{1}^{0}-\mathrm{CA}-$ also $\widehat{\mathbf{P A}}^{\omega} \wedge+\Pi_{1}^{0}-\mathrm{AC}^{0,0} \vdash \Pi_{\infty}^{0}-\mathrm{CA}$ and therefore $\left.\widehat{\mathbf{P A}}^{\omega} \uparrow+\Pi_{1}^{0}-\mathrm{AC}^{0,0} \vdash \mathrm{AC}_{a r}^{0,0}\right)$ and that the interpretation of $\Pi_{1}^{0}-\mathrm{AC}^{0,0}$ uses only $B_{0,1}$ and functionals from $\widehat{\mathbf{P R}}^{\omega}$. Note that the crucial lemma 1 from [28] (restricted to $B_{0,1}$ ) can easily be proved in $\widehat{\mathbf{P R}}^{\omega}+B R_{0,1}$. 
Hence there are functionals $\underline{\tilde{\Psi}} \in \mathcal{F}$ such that

$$
(+)\left\{\begin{aligned}
\mathcal{T} \vdash \forall \underline{\Phi}_{A}, \underline{\Phi}_{(A \rightarrow B)^{p r}}\left(\left(\underline{\Phi}_{A} \text { n.c.i. } A\right)\right. & \wedge\left(\underline{\Phi}_{(A \rightarrow B)^{p r}} \text { n.c.i. }(A \rightarrow B)^{p r}\right) \\
& \left.\rightarrow \forall \underline{h}, \underline{a} B_{0}^{H}\left(\underline{h}, \underline{\tilde{\Psi}}\left(\underline{\Phi}_{A}, \underline{\Phi}_{(A \rightarrow B)^{p r}}, \underline{h}, \underline{a}\right), \underline{a}\right)\right) .
\end{aligned}\right.
$$

Thus $\underline{\Psi}:=\lambda \underline{h} \cdot \underline{\tilde{\Psi}}\left(\underline{\Phi}_{A}, \underline{\Phi}_{(A \rightarrow B)^{p r}}, \underline{h}, \underline{a}\right)$ satisfies the claim made in the proposition.

Remark 3.8 1) Similar to $\underline{\Psi}$ one can also extract $\underline{\xi}, \underline{\zeta} \in \widehat{\mathbf{P R}}^{\omega}+B R_{0,1}$ realizing the universal function quantifiers hidden in ' $\underline{\Phi}_{A}$ n.c.i. $A$ ' and $\underline{\Phi}_{(A \rightarrow B)^{p r}}$ n.c.i. $(A \rightarrow B)^{p r}$ '.

2) In the above proof, (+) can actually be strengthened by not assuming that $\underline{\Phi}_{A}$ (resp. $\left.\underline{\Phi}_{(A \rightarrow B)^{p r}}\right)$ satisfies the no-counterexample interpretation uniformly in the parameters $\underline{a}$ of $A^{8}$, i.e. we can quantify $\underline{a}$ outside the whole implication in $(+)$ and weaken $\left(\underline{\Phi}_{A}\right.$ n.c.i. $\left.A\right)$ (and likewise also $\left(\underline{\Phi}_{(A \rightarrow B)^{p r}}\right.$ n.c.i. $\left.\left.(A \rightarrow B)^{p r}\right)\right)$ to

$$
\forall \underline{h} A_{0}\left(\Phi_{1}^{A} \underline{h}, h_{1}\left(\Phi_{1}^{A} \underline{h}\right), \ldots, \Phi_{k}^{A} \underline{h}, h_{k}\left(\Phi_{1}^{A} \underline{h}, \ldots, \Phi_{k}^{A} \underline{h}\right), \underline{a}\right) .
$$

I.e. we only require $\underline{\Phi}^{A}$ to satisfy the n.c.i. of A for the fixed parameters $\underline{a}$. As in the proof above we now obtain functionals $\underline{\chi}$ which satisfy the modus ponens uniformly in $\underline{h}, \underline{a}$ and functionals $\underline{\Phi}^{A}, \underline{\Phi}^{(A \rightarrow B)^{p r}}$ satisfying the n.c.i. for the parameters $\underline{a}$.

Corollary to the proof of proposition 3.7: The proof above immediately generalizes to the case where $A$ and $B$ contain function parameters $\underline{\alpha}, \underline{\beta}$ and yields functionals $\underline{\Psi}\left(\underline{\Phi}_{A}, \underline{\Phi}_{(A \rightarrow B)^{p r}}, \underline{\alpha}, \underline{\beta}\right)$ which solve the corresponding modus ponens instance uniformly in $\underline{\Phi}_{A}, \underline{\Phi}_{(A \rightarrow B)^{p r}}$ and $\underline{\alpha}, \underline{\beta}$. This in particular implies that we can solve the modus ponens problem uniformly in arbitrary formulas $A, B$ in $\mathcal{L}(\mathbf{P A})$ of fixed quantifier complexities since all formulas $A \in \Pi_{n}^{0}$ can be obtained from $\forall x_{1} \exists y_{1} \ldots \forall x_{n} \exists y_{n}\left(\alpha(\underline{x}, \underline{y})=_{0} 0\right)$ by substituting the characteristic function of the quantifier-free matrix of $A$ (which can be defined in $\widehat{\mathbf{P R}}^{\omega}$ ) for the function variable $\alpha$.

For $A \in \Pi_{3}^{0}, B \in \Pi_{\infty}^{0}$, the functionals $\underline{\Psi} \in \widehat{\mathbf{P R}}^{\omega}+B R_{0,1}$ solving the n.c.i. of the modus ponens rule (which exist by 3.7) can be obtained as the solution of a system of functional equations:

\footnotetext{
${ }^{8}$ Lets assume here for simplicity that $A$ and $B$ contain the same parameters $\underline{a}$. This can be achieved by introducing dummy variables if necessary.
} 
Let $A: \equiv \forall x \exists y \forall z A_{0}(x, y, z)$ and $B: \equiv \forall u_{1} \exists v_{1} B_{0}\left(u_{1}, v_{1}, \ldots\right)$. Consider the following prenex normal form of $A \rightarrow B$

$$
(A \rightarrow B)^{p r}: \equiv \exists x \forall y \exists z \forall u_{1} \exists v_{1} \ldots\left(A_{0}(x, y, z) \rightarrow B_{0}\left(u_{1}, v_{1}, \ldots\right)\right) .
$$

Then

$$
\left((A \rightarrow B)^{p r}\right)^{H} \equiv \forall f, \underline{h} \exists x, z, \underline{v}\left(A_{0}(x, f x, z) \rightarrow B_{0}\left(h_{0} x z, v_{1}, h_{1} x z v_{1}, v_{2}, \ldots\right)\right) .
$$

So the n.c.i. of $(A \rightarrow B)^{p r}$ requires functionals $\Phi_{1}, \Phi_{2}, \Psi_{1}, \Psi_{2}, \ldots$ such that

$$
(*) \forall f, \underline{h}\left(A_{0}\left(\Phi_{1} f \underline{h}, f\left(\Phi_{1} f \underline{h}\right), \Phi_{2} f \underline{h}\right) \rightarrow B_{0}\left(h_{0}\left(\Phi_{1} f \underline{h}, \Phi_{2} f \underline{h}\right), \Psi_{1} f \underline{h}, \ldots\right)\right) .
$$

Since

$$
A^{H} \equiv \forall g, x \exists y A_{0}(x, y, g y),
$$

the n.c.i. of $A$ requires a functional $\Phi_{0}$ such that

$$
(* *) \forall g, x A_{0}\left(x, \Phi_{0} g x, g\left(\Phi_{0} g x\right)\right) \text {. }
$$

To perform a modus ponens using $(*),(* *)$ to obtain a solution for the for the n.c.i. of $B$ we solve the following systems of equations (mp-unification) for $x, f, g$ (uniformly in $\left.\underline{h}, \Phi_{0}, \Phi_{1}, \Phi_{2}\right)$ :

$$
\text { (1) }\left\{\begin{array}{l}
x={ }_{0} \Phi_{1} f \underline{h} \\
f\left(\Phi_{1} f \underline{h}\right)={ }_{0} \Phi_{0} g x \\
\Phi_{2} f \underline{h}={ }_{0} g\left(\Phi_{0} g x\right) .
\end{array}\right.
$$

Let $f[\underline{h}, \underline{\Phi}]$ be the $f$-solution for $\underline{h}, \Phi_{0}, \Phi_{1}, \Phi_{2}$. Taking then $\tilde{h}_{0}:=\lambda x, y . u, \tilde{h}_{i} x y v_{1} \ldots v_{i}:=$ $h_{i} v_{1} \ldots v_{i}($ for $i \geq 1)$ and $\tilde{\Psi}_{i}\left(u, h_{1}, \ldots\right):=\Psi_{i}(f[\underline{\tilde{h}}, \underline{\Phi}], \underline{\tilde{h}})$ we obtain that $\underline{\tilde{\Psi}}$ n.c.i. B.

Remark 3.9 Note that the system of equation (1) is the same as the one resulting from the functional interpretation of the double-negation shift

$$
\forall x^{0} \neg \neg \exists y^{0} \forall z^{0} A_{0}(x, y, z) \rightarrow \neg \neg \forall x \exists y \forall z A_{0}(x, y, z)
$$

solved by Spector [28] using bar recursion in his functional interpretation of classical analysis (via negative translation). For completeness we include here the solution.

In our case it suffices in fact to construct an $f$ such that there exists a $g$ so that (1) holds for $x=\Phi_{1} f \underline{h}$, since the functionals $\underline{\tilde{\Psi}}$ do not depend on $g$. 
In fact we solve (following Spector [28])

$$
\text { (2) } \exists f \forall n \leq \Phi_{1} f \underline{h} \exists g_{n}\left(\Phi_{0}\left(g_{n}, n\right)={ }_{0} f n \wedge g_{n}(f n)={ }_{0} \Phi_{2} f \underline{h}\right)
$$

for $f$. Note that this solves (1) as well: take $x:=n:=\Phi_{1} f \underline{h}$ and $g:=g_{x}$.

Solution of (2): Define

$$
A(f, n): \equiv n \leq \Phi_{1} f \underline{h} \rightarrow \exists g_{n}\left(\Phi_{0}\left(g_{n}, n\right)=f n \wedge g_{n}(f n)=\Phi_{2} f \underline{h}\right) .
$$

We define a functional $B \in 1(0)(1)$ which satisfies

(i) $\forall i<x(B(\overline{f, x} ; x)(i)=f i)$,

(ii) $\forall n \geq x A(B(\overline{f, x} ; x), n)$.

Then $B\left(0^{1}, 0^{0}\right)$ satisfies $\forall n A(B(0,0), n)$, i.e. solves ' $\exists f^{\prime}$ ' in (2).

We now define $B(\overline{f, x} ; x)$ by bar recursion:

Case 1): $\Phi_{1}(\overline{f, x}) \underline{h}<x$. Take $B(\overline{f, x} ; x):=\overline{f, x}$. Then $B(\overline{f, x} ; x)$ trivially satisfies $(i)$ and because of $n \geq x \rightarrow n>\Phi_{1}(\overline{f, x}) \underline{h}$ (by the case) also $(i i)$.

Case 2): $\Phi_{1}(\overline{f, x}) \underline{h} \geq x$. By assumption $B\left(\overline{f, x} *\langle X\rangle ; x^{\prime}\right)$ is defined already such that

(i) ${ }^{\prime} \forall i \leq x\left(B\left(\overline{f, x} *\langle X\rangle ; x^{\prime}\right)(i)=(\overline{f, x} *\langle X\rangle)(i)\right)$ and

(ii) ${ }^{\prime} \forall n \geq x^{\prime} A\left(B\left(\overline{f, x} *\langle X\rangle ; x^{\prime}\right), n\right.$ ) for all $X$ (Note that $\overline{f, x} *\langle X\rangle=\overline{\overline{f, x} *\langle X\rangle, x^{\prime}}$ ).

Define $B(\overline{f, x} ; x):=B\left(\overline{f, x} *\langle K\rangle ; x^{\prime}\right)$,

where $K:=\Phi_{0} g_{x} x$ and $g_{x}:=\lambda X . \Phi_{2}\left(B\left(\overline{f, x} *\langle X\rangle ; x^{\prime}\right)\right) \underline{h}$.

By $(i)^{\prime},(i i)^{\prime}$ we have

$$
\forall n \geq x^{\prime} A(B(\overline{f, x} ; x), n) \text { and } \forall i<x(B(\overline{f, x} ; x)(i)=f i) .
$$

So it remains to show $A(B(\overline{f, x} ; x), x)$, i.e.

$$
\exists g_{x}\left(\Phi_{0}\left(g_{x}, x\right)=B(\overline{f, x} ; x)(x) \wedge g_{x}(B(\overline{f, x} ; x)(x))=\Phi_{2}(B(\overline{f, x} ; x)) \underline{h}\right):
$$

$B(\overline{f, x} ; x)(x)=B\left(\overline{f, x} *\left\langle\Phi_{0} g_{x} x\right\rangle ; x^{\prime}\right)(x) \stackrel{(i)^{\prime}}{=} \Phi_{0}\left(g_{x}, x\right)$.

$g_{x}(B(\overline{f, x} ; x)(x))=\Phi_{2}\left(B\left(\overline{f, x} *\langle B(\overline{f, x} ; x)(x)\rangle ; x^{\prime}\right)\right) \underline{h}=$

$\Phi_{2}\left(B\left(\overline{f, x} *\left\langle\Phi_{0}\left(g_{x}, x\right)\right\rangle ; x^{\prime}\right)\right) \underline{h}=\Phi_{2}(B(\overline{f, x} ; x)) \underline{h}$, which concludes the proof.

We call the system of equations (1) above the mp-system corresponding to $A$ and $A \rightarrow B$.

By the reasoning above we have 
Proposition 3.10 For $A, B \in \mathcal{L}(\mathbf{P A})$ with $A \in \Pi_{3}^{0}, B \in \Pi_{\infty}^{0}$ one can construct functionals $\underline{\Psi} \in \widehat{\mathbf{P R}}^{\omega}+B R_{0,1}$ which uniformly solve the corresponding mp-system.

In a separate paper we intend to investigate in greater generality what types of unification problems can be solved by (restricted forms of) bar recursion.

The use of bar recursion in proposition 3.10 is crucial as the following proposition shows:

Proposition 3.11 Even for $A \in \Pi_{3}^{0}, B \in \Pi_{0}^{0}$ there are no functionals $\underline{\Psi} \in T$ for which it is true in $\mathcal{S}^{\omega}$ that they solve the corresponding mp-system uniformly.

Proof: The mp-system corresponding to $A$ and the unique prenex normal form of $A \rightarrow B$ again is identical to the system of equations emerging from the functional interpretation of the double negation shift

$$
\forall x^{0} \neg \neg \exists y^{0} \forall z^{0} A_{0}(x, y, z) \rightarrow \neg \neg \forall x^{0} \exists y^{0} \forall z^{0} A_{0}(x, y, z) .
$$

So if the mp-system would be solvable in $T$ then this double negation shift and consequently - via negative translation - $\mathbf{P A}^{\omega}+\mathrm{AC}_{a r}^{0,0}$ would have a functional interpretation by functionals in $T$ (verifiable in $\mathcal{S}^{\omega}$ ). However it is known that all $\alpha\left(<\varepsilon_{\varepsilon_{0}}\right)$-recursive functions are provably recursive in $\mathbf{P A}^{\omega}+\mathrm{AC}_{a r}^{0,0}$ whereas the definable functions in $T$ are $\alpha$-recursive with $\alpha<\varepsilon_{0}$ (see e.g. [4]).

In contrast to this result we have

Proposition 3.12 For $A \in \Pi_{2}^{0}, B \in \Pi_{\infty}^{0}$ the corresponding mp-system has a trivial solution by substitution.

Proof: The corresponding system of equations is $x={ }_{0} \Phi_{1} f \underline{h}, \Phi_{0} x=_{0} f x$. Take $f:=\Phi_{0}$ and $x:=\Phi_{1} f \underline{h}$. $\square$.

\section{The pointwise mp-complexity for arbitrary formulas $A, B \in$ $\mathcal{L}(\mathbf{P A})$ and the conditions $(\delta)$ and $(\gamma)$}

In the following we need a slight generalization of a result due to Schwichtenberg $[24],[26]^{9}$ on the closure of $T$ under the rule of bar recursion of type 0 (and 1):

Proposition 4.1 Let $t^{2}\left[\underline{x}^{0}, \underline{h}^{1}\right]$ a term of $T$ containing at most the free variables $\underline{x}$ of type 0 and the variables $\underline{h}$ of type level 1 . Then the functional $\lambda \underline{x}, \underline{h}, z, u, n, y \cdot B_{0, \tau}(t[\underline{x}, \underline{h}], z, u, n, y)$ is definable in $T$ such that $\mathbf{P A}^{\omega}$ (and even $\mathbf{H A}^{\omega}$ ) proves its characterizing equations.

\footnotetext{
${ }^{9}$ Compare also remark 3.1 in [11] for a related result.
} 
Proof: In [26] it is proved that for all closed terms $t, s, r$ of $T$ (of appropriate types) $\lambda n, y \cdot B_{0, \tau}$ trsny is definable in $T$ (formalizable in $\mathbf{H A}^{\omega}$ ). Since there is no restriction on the type $\tau$ we can replace $r, s$ by free variables $z, u$ observing that $B_{0, \tau}$ tzuny $=_{\tau}\left(B_{0, \sigma} \operatorname{trsny}\right) z u$ for suitable closed $\lambda$-terms $r, s$ ( $\sigma$ being a corresponding type). Moreover, Schwichtenberg's proof immediately relativizes uniformly to the case where $t$ is allowed to contain number and function parameters yielding a primitive recursive functional (in the sense of $T$ ) in these parameters and $z, u, n, y$ (to see this one could also use the technique of elimination of free variables from section 5 of $[9])$.

Proposition 4.2 Let $t^{0 \tau_{1} \ldots \tau_{m} \rho_{1} \ldots \rho_{l} \delta_{1} \ldots \delta_{k}}$ a (closed) term of $T_{1}+B R_{0,1}$, where $\delta_{1}=\ldots=\delta_{k}=0, \operatorname{deg}\left(\rho_{1}\right)=\ldots=\operatorname{deg}\left(\rho_{l}\right)=1, \operatorname{deg}\left(\tau_{1}\right), \ldots, \operatorname{deg}\left(\tau_{m}\right) \leq 3$.

Let $\Phi_{1}^{\tau_{1}}, \ldots, \Phi_{m}^{\tau_{m}}$ be closed terms in $T$. Then $s:=\lambda \underline{x}^{\underline{\delta}}, \underline{\underline{h}} \underline{\rho} . t\left(\underline{x}, \underline{h}, \Phi_{1}, \ldots, \Phi_{m}\right)$ is definable as a closed term $\tilde{s}$ in $T$ and $\mathbf{H A}^{\omega}+B R_{0,1} \vdash s=\gamma \tilde{s}$, where $\gamma$ is the type of $s$.

Proof: Let $t[\underline{h}]^{0}$ be built up from $n$-ary function variables $\underline{h}$, the combinators $\Pi, \Sigma$ (of arbitrary finite type) $, 0^{0}, S^{00}$, closed terms $\Phi_{1}^{\tau_{1}}, \ldots, \Phi_{m}^{\tau_{m}} \in T$ with $\operatorname{deg}\left(\tau_{i}\right) \leq 3$ and $B_{0,1}$. We show that $\lambda \underline{h} . t[\underline{h}]$ can be defined in $T$ (note that this proves the proposition since the type level of $R_{1}$ is 3 and $R_{0}$ has type level 2).

For notational simplicity we assume that $\tau_{i}=3$ for $i=1, \ldots, m$. By 'logical normalization' we perform all possible $\Pi, \Sigma$-reductions on ${ }^{10} t[\underline{h}]^{0}$ and denote the result by $\left.\widehat{t} \underline{h}\right]^{0}$ (note that HA $\left.^{\omega} \vdash t[\underline{h}]={ }_{0} \hat{t}[\underline{h}]\right) .{ }^{11}$ The outmost constant or variable of $\widehat{t}[\underline{h}]^{0}$ cannot be $\Pi$ or $\Sigma$ since if $\widehat{t}[\underline{h}]^{0} \equiv \Pi t_{1} t_{2} \ldots t_{i}\left(\right.$ resp. $\left.\Sigma t_{1} t_{2} \ldots t_{j}\right)$ then $i \geq 2$ (resp. $j \geq 3$ ) since $\widehat{t}[\underline{h}]$ is of type 0 . But this contradicts the fact that all possible $\Pi, \Sigma$-reductions have been carried out already. Hence $\widehat{t}[\underline{h}] \equiv 0^{0}, \widehat{t}[\underline{h}] \equiv S(\tilde{t}[\underline{h}]), \widehat{t}[\underline{h}] \equiv \Phi_{i}^{3}\left(t_{0}[\underline{h}]\right), \widehat{t}[\underline{h}] \equiv\left(h_{i}\left(t_{1}[\underline{h}]\right) \ldots\left(t_{j}[\underline{h}]\right)\right)^{0}$ or $\widehat{t}[\underline{h}] \equiv B_{0,1}\left(t_{1}[\underline{h}]\right) \ldots\left(t_{6}[\underline{h}]\right)$. By proposition 4.1 (to be used in the last case only), $\widehat{t}[\underline{h}]$ is primitive recursive (in the sense of $T$ ) in $\underline{h}$ if $\tilde{t}[\underline{h}]$ or $\left(t_{0}[\underline{h}] f_{0}\right)^{0}$ or $\left(t_{1}[\underline{h}]\right)^{0}, \ldots,\left(t_{j}[\underline{h}]\right)^{0}$ resp. $\left(t_{1}[\underline{h}] \underline{f}_{1}\right)^{0}, \ldots,\left(t_{6}[\underline{h}] \underline{f}_{6}\right)^{0}$ are primitive recursive in all of there free variables. Here $\underline{f}_{i}$ are the (possibly empty) tuples of variables needed to reach the ground type 0 (note that the type levels of $f_{0}, \underline{f}_{i}$ are $\leq 1$ since all the arguments of $B_{0,1}$ and $\Phi_{i}^{3}$ have type levels $\leq 2$ ). We now proceed with these terms instead of $t[\underline{h}]$ (note that in the case of $t_{0}[\underline{h}] f_{0}, t_{i}[\underline{h}] \underline{f}_{i}$ we again first have to carry out all possible $\Pi, \Sigma$-reductions since in view of the new arguments $f_{0}, \underline{f}_{i}$ new reductions may be possible). Eventually we end up with terms which no longer contain $B_{0,1}$ and hence are primitive recursive. So $\lambda \underline{h} . t[\underline{h}]$ is a primitive

\footnotetext{
${ }^{10}$ Here we consider the terms $\Phi_{i}^{\tau_{i}}$ as primitives, i.e. we don't carry out $\Pi, \Sigma$-reductions on the $\Pi, \Sigma$ constants occuring in these terms.

${ }^{11}$ Here the notation $s[\underline{h}]$ means that $s$ contains at most free variables from $\underline{h}$.
} 
recursive functional which can be written as a closed term $\tilde{s} \in T$. To see that $\mathbf{H A}^{\omega}+B R_{0,1} \vdash$ $\tilde{s} \underline{h}={ }_{0} t[\underline{h}]$ we argue as follows: Consider a term $r \equiv B_{0,1} t_{1}[\underline{h}] \ldots t_{6}[\underline{h}]$, where $t_{1}[\underline{h}], \ldots, t_{6}[\underline{h}]$ do not contain $B_{0,1}$. By proposition 4.1 we can find a closed term $\tilde{r} \in T$ such that $\tilde{r} \underline{h} n \alpha$ satisfies (provably in $\mathbf{H A}^{\omega}$ ) the instance of $B R_{0,1}$ for $t_{1}[\underline{h}], \ldots, t_{3}[\underline{h}], n, \alpha$. Since $B R_{0,1}$ defines $\lambda n, \alpha \cdot B_{0,1}\left(t_{1}[\underline{h}], \ldots, t_{3}[\underline{h}], n, \alpha\right)$ uniquely in $t_{i}[\underline{h}]$ (provable using extensionality and bar induction or - classically - dependent choice) we have $\tilde{r} \underline{h} n \alpha={ }_{1} B_{0,1}\left(t_{1}[\underline{h}], \ldots, t_{3}[\underline{h}], n, \alpha\right)$ for all $n^{0}, \alpha^{1}$. This can be formalized in e.g. $\mathbf{P A}^{\omega}+\Pi_{\infty}^{0}-\mathrm{DC}_{0}+B R_{0,1}$ (where $\Pi_{\infty}^{0}-\mathrm{DC}_{0}$ is the axiom schema of dependent choice of type 0 for arithmetical predicates) using the facts that all primitive recursive functionals of type 2 are $\mathbf{H A}^{\omega}$-provable extensional (see $[31](2.7 .4))$ and that $=_{1} \in \Pi_{1}^{0}$ is arithmetical. Hence $\mathbf{P A}^{\omega}+\Pi_{\infty}^{0}-\mathrm{DC}_{0}+B R_{0,1} \vdash \tilde{r} \underline{h} n \alpha={ }_{1}$ $B_{0,1}\left(t_{1}[\underline{h}], \ldots, t_{3}[\underline{h}], n, \alpha\right)$. But $\mathbf{P A}^{\omega}+\Pi_{\infty}^{0}-\mathrm{DC}_{0}+B R_{0,1}=\mathbf{P A}^{\omega}+\mathrm{AC}_{a r}^{0,0}+B R_{0,1}=\mathbf{P A}^{\omega}+\Pi_{1}^{0}-$ $\mathrm{AC}^{0,0}+B R_{0,1}$ has a functional interpretation in $\mathbf{H A}^{\omega}+B R_{0,1}$ and hence $\mathbf{H} \mathbf{A}^{\omega}+B R_{0,1} \vdash$ $\tilde{r} \underline{h} n \alpha={ }_{1} B_{0,1}\left(t_{1}[\underline{h}], \ldots, t_{3}[\underline{h}], n, \alpha\right)$. Thus for $\widehat{r} \underline{h}: \equiv \tilde{r}\left(\underline{h}, t_{4}[\underline{h}], t_{5}[\underline{h}], t_{6}[\underline{h}]\right)$ we have $\mathbf{H A}^{\omega}+$ $B R_{0,1} \vdash \widehat{r} \underline{h}={ }_{0} B_{0,1} t_{1}[\underline{h}] \ldots t_{6}[\underline{h}]$. The claim now follows inductively by the normalization argument above using the quantifier-free rule of extensionality of $\mathbf{H A}^{\omega}+B R_{0,1}$.

Remark 4.3 1) Proposition 4.2 is related to a result from [10] (thm.3.2 and remark 1) which in our terminology states that every term (containing only variables type of level $\leq 1)$ of type level $\leq 2$ in $T_{1}+B R_{0,1}$ has computation size strictly less then $\varepsilon_{0}$.

2) Even for closed terms $t^{1}$ proposition 4.2 is false for $\widehat{\mathbf{P R}}^{\omega}+B R_{1,1}$ or $\widehat{\mathbf{P R}}^{\omega}+B R_{0,2}$ instead of $T_{1}+B R_{0,1}$ : the system $\widehat{\mathbf{P A}}^{\omega} \wedge+\Sigma_{1}^{1}-\mathrm{DC}$ has ${ }^{12}$ (via negative translation) a functional interpretation in $\widehat{\mathbf{P R}}^{\omega}+B R_{1,1}$. But the system is proof-theoretically stronger than PA (see e.g. [2] pp. 128-129) and proves more recursive functions to be total than are definable in $T$. The counterexample for $\widehat{\mathbf{P R}}^{\omega}+B R_{0,2}$ follows from the fact that $B R_{1,1}$ can be reduced to $B R_{0,2}$ (see [18],[12]). The essential formal difference between $B R_{0,1}$ and both $B R_{1,1}, B R_{0,2}$ is that the corresponding bar recursor constant $B_{0,1}$ is of type level 3 whereas both $B_{1,1}$ and $B_{0,2}$ are of type level 4 (see also [10], appendix 2).

3) Even for closed terms $t^{1}$ proposition 4.2 is false for $T_{2}+B R_{0,1}$ instead of $T_{1}+B R_{0,1}$. This follows from the fact that $R_{\rho}$ with $\operatorname{deg}(\rho)=2$ (which has type level 4) can be used to iterate $B_{0,1}$ as a type-3-level functional which goes beyond $\alpha\left(<\varepsilon_{0}\right)$-recursion. In fact $T_{2}+B R_{0,1}$ corresponds to $\mathcal{T}_{3,4}$ in [10] where it is shown that the computation size of terms in $\mathcal{T}_{3,4}$ is $<\varepsilon_{\omega^{\omega}}$ and that this is optimal.

\footnotetext{
${ }^{12}$ Here $\Sigma_{1}^{1}$-DC denotes the schema of dependent choice of type 1 restricted to $\Sigma_{1}^{1}$-formulas.
} 
Corollary 4.4 1) The same functionals of type level $\leq 2$ are definable in $T$ and in $\widehat{\mathbf{P R}}^{\omega}+B R_{0,1}$ (but there union $T+B R_{0,1}$ allows to define more functions). ${ }^{13}$

2) Let $\widehat{\mathbf{P A}}_{1}^{\omega} \backslash$ be the extension of $\widehat{\mathbf{P A}}^{\omega} \wedge$ obtained by adding the Gödel recursor $R_{1}$ for type1-recursion with its axioms. Let $\mathcal{A}:=\widehat{\mathbf{P A}}_{1}^{\omega} \uparrow+A C_{a r}^{0,0}+A C$-qf. If $\mathcal{A} \vdash \forall x^{\rho} \exists y^{\tau} A_{0}(x, y)$, where $\operatorname{deg}(\rho) \leq 1, \operatorname{deg}(\tau) \leq 2$ and $A_{0}(x, y)$ quantifier-free with only $x, y$ as free variables, then one can extract a closed term $t \in T$ such that

$$
\mathcal{S}^{\omega} \models \forall x^{\delta} A_{0}(x, t x)
$$

3) Besides the usual functional interpretation (combined with negative translation) of PA in $T$, PA also has - via PA $\subset \widehat{\mathbf{P A}}^{\omega} \Lambda+A C_{a r}^{0,0}-$ a functional interpretation in $\widehat{\mathbf{P R}}^{\omega}+B R_{0,1}$. Both functional interpretations are faithful w.r.t. the provably recursive functions of $\mathbf{P A}$ whereas the interpretation in their union $T+B R_{0,1}$ is not.

Proof: 1) By proposition 4.2, every definable functional of type level $\leq 2$ is definable in $T$. The other direction follows from the facts that the definable function(al)s of types 0 and 1 in $T$ are just the $\alpha\left(<\varepsilon_{0}\right)$-recursive ones, that all $\alpha\left(<\varepsilon_{0}\right)$-recursive function(al)s of type level $\leq 2$ are provably recursive in $\widehat{\mathbf{P A}}^{\omega} \uparrow+\mathrm{AC}_{a r}^{0,0}$ (since the extension $\mathbf{P A}^{+}$of $\mathbf{P A}$ by function parameters is a subsystem of $\widehat{\mathbf{P A}}^{\omega} \uparrow+\mathrm{AC}_{\text {ar }}^{0,0}$ ) and that this system has (via negative translation) a functional interpretation in $\widehat{\mathbf{P R}}^{\omega}+B R_{0,1}$ (see the proof of proposition 3.7). 2) From the fact that $\mathcal{A}$ has (via negative translation) a functional interpretation in $T_{1}+$ $B R_{0,1}$ (see again the proof of proposition 3.7) and proposition 4.2 it follows that $\mathbf{H A}^{\omega}+$ $B R_{0,1} \vdash \forall x^{\delta} A_{0}(x, t x)$ for some closed $t \in T$. The type structure of all continuous settheoretical functionals $\mathbf{C}$ from [23] (called $\mathbf{S}$ by Scarpellini) is a model of $\mathbf{H A}^{\omega}+B R_{0,1}$. The conclusion now follows from the facts that $\mathbf{C}_{0}=\mathcal{S}_{0}$ and $\mathbf{C}_{1}=\mathcal{S}_{1}$ and that $\forall f \in$ $\omega^{\omega}\left([\Phi]_{\mathbf{C}} f=[\Phi]_{\mathcal{S}} f\right)$ for all closed terms $\Phi \in T$ of type 2 .

3) follows from the proof of 2).

Using proposition 3.7 and proposition 4.2 we obtain that $\mathbf{P A}{ }^{\omega}, T$ suffices for a pointwise n.c.i. of the modus ponens rule:

Proposition 4.5 Let $A, B$ be prenex formulas in $\mathcal{L}(\mathbf{P A})$ and $(A \rightarrow B)^{p r}$ some prenex normal form of $A \rightarrow B$. Then there are functionals $\underline{\chi} \in \widehat{\mathbf{P R}}^{\omega}+B R_{0,1}$ such that:

\footnotetext{
${ }^{13}$ Note that each closed term $t^{2} \in \widehat{\mathbf{P R}}^{\omega}+B R_{0,1}$ represents a functional in $\mathcal{S}^{\omega}$ (so that the comparison with the type-2-functionals definable in $T$ makes sense). This can be seen e.g. by interpreting $t$ in the model of all continuous set-theoretical functionals $\mathbf{C}$ from [23] since $\mathbf{C}_{1}=\omega^{\omega}$.
} 
If $\underline{\Phi}_{A}, \underline{\Phi}_{(A \rightarrow B)^{p r}} \in \mathcal{S}^{\omega}$ are continuous ${ }^{14}$ then also $\underline{\chi}\left(\underline{\Phi}_{A}, \underline{\Phi}_{(A \rightarrow B)^{p r}}\right) \in \mathcal{S}^{\omega}$ are continuous and if in addition

$$
\text { (1) } \mathcal{S}^{\omega} \models\left(\underline{\Phi}_{A} \text { n.c.i.A }\right) \wedge\left(\underline{\Phi}_{(A \rightarrow B)^{p r}} \text { n.c.i. }(A \rightarrow B)^{p r}\right) \text {, }
$$

then

$$
\text { (2) } \mathcal{S}^{\omega} \models \underline{\chi}\left(\underline{\Phi}_{A}, \underline{\Phi}_{(A \rightarrow B)^{p r}}\right) \text { n.c.i. B. }
$$

Furthermore if $\underline{\Phi}_{A}, \underline{\Phi}_{(A \rightarrow B)^{p r}}$ are closed terms of $T$ then $\underline{\chi}\left(\underline{\Phi}_{A}, \underline{\Phi}_{(A \rightarrow B)^{p r}}\right)$ can effectively be written as functionals in $T$.

As in proposition 3.7 this generalizes to the case where $A, B$ contain function parameters $\underline{\alpha}, \underline{\beta}$ yielding $\underline{\chi}$ as functionals in $\underline{\Phi}_{A}, \underline{\Phi}_{(A \rightarrow B)^{p r}}, \underline{\alpha}, \underline{\beta}$ with $\lambda \underline{\alpha}, \underline{\beta} \cdot \underline{\chi}\left(\underline{\Phi}_{A}, \underline{\Phi}_{(A \rightarrow B)^{p r}}, \underline{\alpha}, \underline{\beta}\right) \in T$ if $\underline{\Phi}_{A}, \underline{\Phi}_{(A \rightarrow B)^{p r}} \in T$.

Proof: The first part follows from proposition 3.7 using the fact that the extensional type structure $\mathbf{C}$ of all continuous functionals from [23] (denoted by $\mathbf{S}$ in [23]) is a model of $\mathbf{P A}^{\omega}+B R_{0,1}, \mathbf{C}_{1}=\mathcal{S}_{1}, \mathbf{C}_{2} \subset \mathcal{S}_{2}$ and the fact that ' $\underline{\Phi}_{A}, \underline{\Phi}_{(A \rightarrow B)^{p r}} \in \mathcal{S}^{\omega}$ continuous' iff ' $\underline{\Phi}_{A}, \underline{\Phi}_{(A \rightarrow B)^{p r}} \in \mathbf{C}$ ' since the type levels of these functionals are $\leq 2$.

The second part follows using proposition 4.2

Remark 4.6 Note that for every $\mathbb{N}$-true prenex formula $A \in \mathcal{L}(\mathbf{P A})$ there are always continuous functionals $\underline{\Phi}_{A} \in \mathcal{S}^{\omega}$ satisfying the n.c.i. of $A$ : apply unbounded search to find the least $\left\langle x_{1}, \ldots, x_{k}\right\rangle$ such that $A_{0}\left(x_{1}, h_{1} x_{1}, \ldots\right)$. Furthermore by bounded search one can construct uniformly in $\underline{\Phi}_{A}$ functionals $\underline{\Phi}_{A}^{*}$ such that the implication

$$
\left(\underline{\Phi}_{A} \text { n.c.i. } A\right) \Rightarrow\left(\underline{\Phi}_{A}^{*} \text { n.c.i } A\right) \wedge\left(\underline{\Phi}_{A}^{*} \text { are continuous }\right)
$$

holds for all $\underline{\Phi}_{A} \in \mathcal{S}^{\omega}$.

Proposition 4.5 implies the following result (which does not follow from the approaches to the no-counterexample interpretation via cut-elimination or functional interpretation)

Corollary 4.7 The n.c.i. of PA in $T$ (or -equivalently - by all $\alpha\left(<\varepsilon_{0}\right)$-recursive functionals) satisfies Kreisel's condition $(\delta)$ in the following sense: Let $A, B \in \mathcal{L}(\mathbf{P A})$ be prenex. If

$\underline{\Phi}_{A}$ n.c.i. $A$ is true for some tuple of closed terms $\underline{\Phi}_{A} \in T$

and

$$
\mathbf{P A} \vdash A \rightarrow B,
$$

then one can construct $\underline{\Phi}_{B} \in T$ such that

$\underline{\Phi}_{B}$ n.c.i. $B$ is true.

\footnotetext{
${ }^{14}$ Here we assume that $\underline{\Phi}_{A}, \underline{\Phi}_{(A \rightarrow B)^{p r}}$ have the appropriate types to make them candidates for the n.c.i. of $A$ resp. $(A \rightarrow B)^{p r}$.
} 
Proof: By functional interpretation one extract $\underline{\Phi}_{(A \rightarrow B)^{p r}} \in T$ such that

$$
\mathbf{P A}^{\omega} \vdash \underline{\Phi}_{(A \rightarrow B)^{p r}} \text { n.c.i. }(A \rightarrow B)^{p r},
$$

where $(A \rightarrow B)^{p r}$ is any prenex normal form of $A \rightarrow B$. The corollary now follows from 4.5. $\square$.

Remark 4.8 By inspecting carefully the instances of $B R_{0,1}$ used in the proof of proposition 3.7 for given $\underline{\Phi}_{A}, \underline{\Phi}_{(A \rightarrow B)^{p r}} \in T$ and using the fact that proposition 4.1 can be formalized in $\mathbf{H A}^{\omega}$ there should be no problem to obtain corollary 4.7 also as a rule w.r.t. to $\mathbf{P A}^{\omega}$ provability, i.e.

$$
\mathbf{P A}^{\omega} \vdash \underline{\Phi}_{A} \text { n.c.i. } A \Rightarrow \mathbf{P A}^{\omega} \vdash \underline{\Phi}_{B} \text { n.c.i. } B\left(\text { even } \mathbf{H A}^{\omega} \vdash \underline{\Phi}_{B} \text { n.c.i. B }\right) .
$$

However we will not spell out the details here.

In [17], Kreisel gives a definition of an 'interpretation of a theory $\mathcal{T}$ in a constructive system $F^{\prime}$ which essentially replaces the condition $(\delta)$ from his previous definition in [15] by the requirement

$\left(\delta^{\prime}\right)$ : the interpretation $A_{n}$ of $A$ implies $A$ logically.

Kreisel mentions in his discussion in remark 2.2 of [17] that this condition is satisfied for the n.c.i. of predicate logic as well as of PA only if 'logically' is understood in the sense of (classical) second-order logic, i.e. first-order logic extended by function quantifier and the axiom of choice schema

$$
\forall x \exists y A(x, y) \rightarrow \exists f \forall x A(x, f x) .
$$

$(\delta)$ does not imply $\left(\delta^{\prime}\right)$ since the trivial interpretation mentioned in [15](pp.248-249) satisfies $(\delta)$ but not $\left(\delta^{\prime}\right)$. In the other direction $\left(\delta^{\prime}\right)$ does not imply $(\delta)$ either. E.g. the n.c.i. of $\mathbf{P A}_{n+1}$ in $T_{n}$ trivially satisfies $\left(\delta^{\prime}\right)$ (again in the sense of second-order logic) but does not satisfy $(\delta)$ by corollary 2.4 above.

In [19] Kreisel formulates both $\left(\delta^{\prime}\right)$ and a version of $(\delta)$ which reads as follows

'Having made a guess at $A^{*}$, which, in the case of the no-counterexample interpretation above is $A_{0}(F, f)$, we try to find, for each axiom $A$, a functional $s_{A}$ such that

$$
\mathcal{P} \vdash A^{*}\left(s_{A}, t\right) \text { for variable } t,
$$

and for each rule of inference, deriving $A$ from $\bar{A}$ and $\overline{\bar{A}}$ say, a functor $\Phi_{A}$ such that $\mathcal{P} \vdash A^{*}\left[\Phi_{A}\left(s_{1}, s_{2}\right), t\right]$ holds provided both $\mathcal{P} \vdash \bar{A}^{*}\left(s_{1}, t_{1}\right)$ and $\mathcal{P} \vdash \overline{\bar{A}}^{*}\left(s_{2}, t_{2}\right)$ hold (for variables $t_{1}$ and $t_{2}$ of appropriate type)' (p. 378).

This is stronger than the previous formulation of $(\delta)$ from [15] by requiring the existence of functors which perform the rules pointwise (for provably correct interpretations for $\bar{A}$ 
and $\overline{\bar{A}}$ ) but also weaker by assuming the $\mathcal{P}$-provability of these interpretations. However the latter does not change the failure of the condition for the n.c.i. of $\mathbf{P A} \mathbf{A}_{n+1}$ in $T_{n}$.

Finally we consider Kreisel's condition $(\gamma)$ from his definition of an interpretation as given in [15]. For the n.c.i. of PA in $T$ this condition spells out as follows

$(\gamma)\left\{\begin{array}{l}\text { If } A \equiv \exists x_{1} \forall y_{1} \ldots \exists x_{k} \forall y_{k} A_{0}\left(x_{1}, y_{1}, \ldots, x_{k}, y_{k}, \underline{a}\right) \in \mathcal{L}(\mathbf{P A}) \text { and PA } \vdash \neg A . \\ \text { Then constructively it holds that for all functionals } \underline{\Phi} \in T \text { (of suitable types) } \\ \text { there are } \underline{h} \text { such that } A_{0}\left(\Phi_{1} \underline{h}, h_{1}\left(\Phi_{1} \underline{h}\right), \ldots, \Phi_{k} \underline{h}, h_{k}\left(\Phi_{1} \underline{h}, \ldots, \Phi_{k} \underline{h}\right), \underline{a}\right) \text { is false. }\end{array}\right.$

Similar to $(\delta)$ also the condition $(\gamma)$ does not follow from the approach to the n.c.i. via cut-elimination or functional interpretation. In [16] Kreisel gave a complicated proof of $(\gamma)$ using again the method of $\varepsilon$-substitution from [1]. We now prove a new uniform version of $(\gamma)$ :

Proposition 4.9 Let $A: \equiv \exists x_{1} \forall y_{1} \ldots \exists x_{k} \forall y_{k} A_{0}\left(x_{1}, y_{1}, \ldots, x_{k}, y_{k}, \underline{a}\right) \in \mathcal{L}(\mathbf{P A})$ and PA $\vdash$ $\neg A$. Then from the proof of $\neg A$ one can extract functionals $\underline{\chi} \in \widehat{\mathbf{P R}}^{\omega}+B R_{0,1}$ such that

$$
\widehat{\mathbf{H A}}^{\omega} \wedge+B R_{0,1} \vdash \forall \underline{\Phi} \neg A_{0}\left(\Phi_{1} \underline{h}, h_{1}\left(\Phi_{1} \underline{h}\right), \ldots, \Phi_{k} \underline{h}, h_{k}\left(\Phi_{1} \underline{h}, \ldots, \Phi_{k} \underline{h}\right), \underline{a}\right),
$$

where $\underline{h}:=\underline{\chi}(\underline{a}, \underline{\Phi})$.

Moreover for $\underline{\Phi} \in T$ (and $\widehat{\mathbf{H A}}^{\omega} \wedge$ replaced by $\mathbf{H A}^{\omega}$ ) one can construct a function term $t_{\Phi} \in T$ such that $\underline{\chi}(\underline{a}, \underline{\Phi})$ can be replaced by $\underline{\tilde{\chi}}(\underline{a})$, where $\tilde{\chi}_{i}(\underline{a}):=\lambda n \cdot\left(\overline{\chi_{i}(\underline{a}, \underline{\Phi})}\left(t_{\underline{\Phi}}(\underline{a}, \underline{\chi}(\underline{a}, \underline{\Phi}))\right)\right)_{n}$ and $\lambda \underline{a}, n \cdot\left(\overline{\chi_{i}(\underline{a}, \underline{\Phi})}\left(t_{\underline{\Phi}}(\underline{a}, \underline{\chi}(\underline{a}, \underline{\Phi}))\right)\right)_{n} \in T \cdot{ }^{15}$

Proof: As in the proof of proposition 3.7 one has

$$
\widehat{\mathbf{P A}}^{\omega} \wedge+\mathrm{AC}_{a r}^{0,0} \vdash \exists \underline{\Phi} \forall \underline{h} A_{0}\left(\Phi_{1} \underline{h}, h_{1}\left(\Phi_{1} \underline{h}\right), \ldots, \Phi_{k} \underline{h}, h_{k}\left(\Phi_{1} \underline{h}, \ldots, \Phi_{k} \underline{h}\right), \underline{a}\right) \rightarrow A .
$$

Hence - using PA $\vdash \neg A$ and the fact that $\mathbf{P A} \subset \widehat{\mathbf{P A}}^{\omega} \wedge+\mathrm{AC}_{a r}^{0,0}-$ one has

$$
\widehat{\mathbf{P A}}^{\omega} \wedge+\mathrm{AC}_{a r}^{0,0} \vdash \forall \underline{a}, \underline{\Phi} \exists \underline{h} \neg A_{0}\left(\Phi_{1} \underline{h}, h_{1}\left(\Phi_{1} \underline{h}\right), \ldots, \Phi_{k} \underline{h}, h_{k}\left(\Phi_{1} \underline{h}, \ldots, \Phi_{k} \underline{h}\right), \underline{a}\right) .
$$

The first part of the proposition now follows from the fact that $\widehat{\mathbf{P A}}^{\omega} \uparrow+\mathrm{AC}_{\text {ar }}^{0,0}$ has (via negative translation) a functional interpretation in $\widehat{\mathbf{H A}}^{\omega} \wedge+B R_{0,1}$.

\footnotetext{
${ }^{15}$ Here we refer to a standard coding $\bar{f} x:=\langle f 0, \ldots, f(x-1)\rangle$ of finite sequences.
} 
For the second part we use that for $\underline{\Phi} \in T$ there is a function term $t_{A} \in T$ such that

$$
t_{A} \underline{a} \underline{h}={ }_{0} 0 \leftrightarrow \neg A_{0}\left(\Phi_{1} \underline{h}, h_{1}\left(\Phi_{1} \underline{h}\right), \ldots, \Phi_{k} \underline{h}, h_{k}\left(\Phi_{1} \underline{h}, \ldots, \Phi_{k} \underline{h}\right), \underline{a}\right) .
$$

By $[31](2.7 .8) t_{A}$ is continuous in $\underline{h}$, provably in $\mathbf{H A}^{\omega}$ with a modulus of pointwise continuity $t \in T$, i.e.

$$
\forall \underline{h}, \underline{\tilde{h}}\left(\bigwedge_{i}\left(\overline{h_{i}}(t(\underline{a}, \underline{h}))={ }_{0} \overline{\tilde{h}}_{i}(t(\underline{a}, \underline{h})) \rightarrow t_{A}(\underline{a}, \underline{h})={ }_{0} t_{A}(\underline{a}, \underline{\tilde{h}})\right) .\right.
$$

The last claim in the proposition follows from 4.2. $\square$.

Remark 4.10 Classically one can easily find functions $\underline{h}$ satisfying $(\gamma)$ for $\underline{\Phi} \in T$ (or for arbitrary continuous $\Phi$ ) by unbounded recursive search:

Let $\underline{\Phi} \in T$. Using the continuity argument from the proof above $\exists \underline{h} \neg A_{0}(\ldots \underline{h} \ldots)$ (which classically follows trivially from the assumptions of $(\gamma)$ as in the proof above) implies $\exists \underline{x}^{0} \neg A_{0}\left(\ldots \lambda n .\left(x_{1}\right)_{n}, \ldots, \lambda n .\left(x_{k}\right)_{n} \ldots\right)$. By unbounded search (recursively in $\left.\underline{a}\right)$ one finds $\underline{x}$ realizing $\exists \underline{x}$.

\section{References}

[1] Ackermann, W., Zur Widerspruchsfreiheit der reinen Zahlentheorie. Mathematische Annalen 117, pp. 162-194 (1940).

[2] Buchholz, H., Feferman, S., Pohlers, W., Sieg, W., Iterated Inductive Definitions and Subsystems of Analysis: Recent Proof-Theoretical Studies. Springer LNM 897, Springer, Berlin Heidelberg New York, 1981.

[3] Damnjanovic, Z., Minimal realizability. J. Symbolic Logic 60, pp. 1208-1241 (1995).

[4] Feferman, S., Theories of finite type related to mathematical practice. In: Barwise, J. (ed.), Handbook of Mathematical Logic, North-Holland, Amsterdam, pp. 913-972 (1977).

[5] Feferman, S., Kreisel's 'Unwinding Program'. In: P. Odifreddi (ed.), Kreiseliana: about and around Georg Kreisel, A.K. Peters, Wellesley Massachusetts, pp. 247-273 (1996).

[6] Gödel, K., Vortrag bei Zilsel (1938). With English translation in [8], pp. 86-113.

[7] Gödel, K., Über eine bisher noch nicht benütze Erweiterung des finiten Standpunktes. Dialectica 12, pp. 280-287 (1958).

[8] Gödel, K., Collected Works, Vol. III. Unpublished Essays and Lectures. Oxford University Press, New York 1995. 
[9] Howard, W.A., Functional interpretation of bar induction by bar recursion. Compositio Mathematica 20, pp. 107-124 (1968).

[10] Howard, W.A., Ordinal analysis of simple cases of bar recursion. J. Symbolic Logic 46, pp. 17-30 (1981).

[11] Howard, W.A., Ordinal analysis of bar recursion of type zero. Compositio Mathematica 42, pp. 105-119 (1981).

[12] Howard, W.A., Kreisel, G., Transfinite induction and bar induction of types zero and one, and the role of continuity in intuitionistic analysis. J. Symbolic Logic 31, pp. 325-358 (1966).

[13] Kleene, S.C., Recursive functionals and quantifiers of finite types I. Trans. Amer. Math. Soc. 91, pp. 1-52 (1959).

[14] Kohlenbach, U., A note on the paper 'Minimal Realizability' by Z. Damnjanovic (JSL 60, pp. 1208-1241,1995) (in preparation).

[15] Kreisel, G., On the interpretation of non-finitist proofs, part I. J. Symbolic Logic 16, pp.241-267 (1951).

[16] Kreisel, G., On the interpretation of non-finitist proofs, part II: Interpretation of number theory, applications. J. Symbolic Logic 17, pp. 43-58 (1952).

[17] Kreisel, G., Mathematical significance of consistency proofs. J. Symbolic Logic 23, pp. 155-182 (1958).

[18] Kreisel, G., Functions, ordinals, species. In: Proc. 3rd Int. Congr. Amsterdam, pp. 145-159 (1968).

[19] Kreisel, G., A survey of proof theory. J. Symbolic Logic 33, pp. 321-388 (1968).

[20] Kreisel, G., Levy, A., Reflection principles and their use for establishing the complexity of axiomatic systems. Z. Math. Logik Grundlagen Math. 14, pp. 97-142 (1968).

[21] Parsons, C., Proof-theoretic analysis of restricted induction schemata. J. Symbolic Logic 36, p. 361 (1971).

[22] Parsons, C., On n-quantifier induction. J. Symbolic Logic 37, pp. 466-482 (1972).

[23] Scarpellini, B., A model for bar recursion of higher types. Compositio Mathematica 23, pp. 123-153 (1971). 
[24] Schwichtenberg, H., Einige Anwendungen von unendlichen Termen und Wertfunktionalen. Habilitationsschrift, Münster (1973).

[25] Schwichtenberg, H., Proof theory: some applications of cut-elimination. In: Barwise, J. (ed.), Handbook of Mathematical Logic, North-Holland, Amsterdam, pp. 867-895 (1977).

[26] Schwichtenberg, H., On bar recursion of types 0 and 1. J. Symbolic Journal 44, pp. 325-329 (1979).

[27] Sieg, W., Parsons, C., Introductory note to *1938a ('Vortrag bei Zilsels'). In: [8], pp. $62-85$.

[28] Spector, C., Provably recursive functionals of analysis: a consistency proof of analysis by an extension of principles formulated in current intuitionistic mathematics. In: Recursive function theory, Proceedings of Symposia in Pure Mathematics, vol. 5 (J.C.E. Dekker (ed.)), AMS, Providence, R.I., pp. 1-27 (1962).

[29] Tait, W.W., The substitution method. J. Symbolic Logic 30, pp. 175-192 (1965).

[30] Tait, W.W., Normal derivability in classical logic. In: Barwise, J. (ed.), The syntax and Semantics of Infinitary Languages, Springer, Berlin, pp. 204-236 (1968).

[31] Troelstra, A.S. (ed.) Metamathematical investigation of intuitionistic arithmetic and analysis. Springer Lecture Notes in Mathematics 344 (1973). 


\section{Recent BRICS Report Series Publications}

RS-97-42 Ulrich Kohlenbach. On the No-Counterexample Interpretation. December 1997. 26 pp.

RS-97-41 Jon G. Riecke and Anders B. Sandholm. A Relational Account of Call-by-Value Sequentiality. December 1997. 24 pp. Appears in Twelfth Annual IEEE Symposium on Logic in Computer Science, LICS '97 Proceedings, pages 258-267.

RS-97-40 Harry Buhrman, Richard Cleve, and Wim van Dam. Quantum Entanglement and Communication Complexity. December 1997. 14 pp.

RS-97-39 Ian Stark. Names, Equations, Relations: Practical Ways to Reason about 'new'. December 1997. $\mathrm{ii}+33$ pp. This supersedes the earlier BRICS Report RS-96-31. It also expands on the paper presented in Groote and Hindley, editors, Typed Lambda Calculi and Applications: 3rd International Conference, TLCA '97 Proceedings, LNCS 1210, 1997, pages 336-353.

RS-97-38 Michał Hańćkowiak, Michał Karoński, and Alessandro Panconesi. On the Distributed Complexity of Computing Maximal Matchings. December 1997. 16 pp. To appear in The Ninth Annual ACM-SIAM Symposium on Discrete Algorithms, SODA 98.

RS-97-37 David A. Grable and Alessandro Panconesi. Fast Distributed Algorithms for Brooks-Vizing Colourings (Extended Abstract). December 1997. 20 pp. To appear in The Ninth Annual ACMSIAM Symposium on Discrete Algorithms, SODA '98.

RS-97-36 Thomas Troels Hildebrandt, Prakash Panangaden, and Glynn Winskel. Relational Semantics of Non-Deterministic Dataflow. December 1997. 21 pp.

RS-97-35 Gian Luca Cattani, Marcelo P. Fiore, and Glynn Winskel. A Theory of Recursive Domains with Applications to Concurrency. December 1997. ii+23 pp.

RS-97-34 Gian Luca Cattani, Ian Stark, and Glynn Winskel. Presheaf Models for the $\pi$-Calculus. December 1997. ii+27 pp. Appears in Moggi and Rosolini, editors, Category Theory and Computer Science: 7th International Conference, CTCS '97 Proceedings, LNCS 1290, 1997, pages 106-126. 